\title{
Chronic COVID-19 / Chronic Fatigue Syndrome (ME/CFS) following the first pandemic wave in Germany and biomarkers associated with symptom severity
}

Claudia Kedor ( $\square$ claudia.kedor@charite.de)

Charité Universitätsmedizin Berlin https://orcid.org/0000-0001-9361-9213

Helma Freitag

Charité Universitätsmedizin Berlin

Lil Meyer-Arndt

Charité Universitätsmedizin Berlin

Kirsten Wittke

Charité Universitätsmedizin Berlin

Leif Hanitsch

Charité Universitätsmedizin Berlin

Thomas Zoller

Charite Hospital https://orcid.org/0000-0002-9797-1692

\section{Fridolin Steinbeis}

Charité Universitätsmedizin Berlin

\section{Haffke Milan}

Charité Universitätsmedizin Berlin

\section{Gordon Rudolf}

Charité Universitätsmedizin Berlin

\section{Bettina Heidecker}

Charite University Hospital Berlin

Thomas Bobbert

Joachim Spranger

https://orcid.org/0000-0002-8900-4467

Harald Heidecke

Celltrend $\mathrm{GmbH}$

Hans-Dieter Volk

Charité - Universitätsmedizin Berlin https://orcid.org/0000-0002-7743-6668

Carsten Skurk

Charite Universitätsmedizin Berlin

Friedemann Paul 
Charité

\section{Uta Behrends}

Technical University of Munich

Judith Bellmann-Strobl

Charité https://orcid.org/0000-0003-2615-1643

\section{Carmen Scheibenbogen}

Institute for Medical Immunology, Charité University Hospital Berlin, Campus Virchow

\section{Article}

Keywords: Long-lasting Symptoms, Prospective Observational Cohort Study, Exertion Intolerance, Hand Grip Strength, Inflammation, Hypoperfusion

Posted Date: April 9th, 2021

DOl: https://doi.org/10.21203/rs.3.rs-384347/v1

License: (c) (1) This work is licensed under a Creative Commons Attribution 4.0 International License. Read Full License

Version of Record: A version of this preprint was published at Nature Communications on August 30th, 2022. See the published version at https://doi.org/10.1038/s41467-022-32507-6. 


\section{TITLE PAGE}

\section{Chronic COVID-19 / Chronic Fatigue Syndrome (ME/CFS) following the first pandemic wave in Germany and biomarkers associated with symptom severity}

Kedor $\mathrm{C}^{1+\star}$, Freitag $\mathrm{H}^{1+}$, Meyer-Arndt $\mathrm{L}^{2}$, Wittke $\mathrm{K}^{1}$, Hanitsch $\mathrm{LG}^{1}$, Zoller $\mathrm{T}^{3}$, Steinbeis $\mathrm{F}^{3}$, Haffke $M^{1}$, Rudolf $G^{1}$, Heidecker $B^{4}$, Bobbert $T^{5}$, Spranger $J^{5}$, Heidecke $H^{6}$, Volk $H^{1,5}$, Skurk $C^{4}$, Paul $\mathrm{F}^{2}$, Behrends $\mathrm{U}^{8,9,10}$, Bellmann-Strobl $\mathrm{J}^{2}$, Scheibenbogen $\mathrm{C}^{1}$

* corresponding author

${ }^{+}$shared authorships

${ }^{1}$ Charité - Universitätsmedizin Berlin, corporate member of Freie Universität Berlin, Humboldt Universität zu Berlin, Berlin Institute of Health, and Institute of Medical Immunology, Berlin, Germany,

${ }^{2}$ Charité - Universitätsmedizin Berlin, corporate member of Freie Universität Berlin, Humboldt Universität zu Berlin and Experimental and Clinical Research Center, Max Delbrueck Center for Molecular Medicine, Berlin, Germany

${ }^{3}$ Department of Infectious Diseases and Respiratory Medicine, Berlin, Charité

${ }^{4}$ Department of Cardiology, Berlin, Germany, Berlin, Charité

${ }^{5}$ Department of Endocrinology, Berlin, Germany, Berlin, Charité

${ }^{6}$ Celltrend, Luckenwalde

${ }^{7}$ Berlin Institute of Health, Center for Regenerative Therapies (BCRT), Berlin, Germany

${ }^{8}$ Technical University of Munich, School of Medicine, Childrens' Hospital

${ }^{9}$ German Center for Infection Research (DZIF)

${ }^{10}$ Helmholtz Center Munich (HMGU), AGV Research Unit Gene Vectors

Contact information:

e-mail: claudia.kedor@charite.de,

Telephone: +4930450624354

FAX: +4930450524962

Adress: Augustenburger Platz 1, 13353 Berlin 


\section{Abstract}

A subset of patients has long-lasting symptoms after mild to moderate COVID-19. In a prospective observational cohort study we analysed clinical and laboratory parameters in 42 patients (29 female/13 male, median age 36.5 years) with persistent moderate to severe fatigue and exertion intolerance six months following COVID-19 referred to as Chronic COVID-19 Syndrome (CCS). Most patients were moderately to severely impaired in daily live. 19 patients fulfilled the 2003 Canadian Consensus Criteria for myalgic encephalomyelitis/chronic fatigue syndrome referred to as CFS/CCS. Hand grip strength (HGS) was diminished in most patients. Association of several biomarker with key symptoms of physical and mental fatigue and post exertional malaise indicate low level inflammation and hypoperfusion as potential pathomechanisms.

Infection with severe acute respiratory syndrome coronavirus type 2 (SARS-CoV-2) poses a major threat for developing chronic morbidity. While older patients or those with risk factors have a high risk of severe or critical COVID-19 (Corona Virus Disease) mortality and morbidity, in about $80 \%$ of cases COVID-19 is mild according to WHO criteria. Soon there were reports, however, of patients with persistent symptoms following mild COVID-19 referred to as long COVID. ${ }^{1,2}$ Frequent symptoms included fatigue, impaired physical and cognitive function, headache, breathlessness, palpitations and many other symptoms, impairing activities of daily living in many patients. ${ }^{3-8} \mathrm{~A}$ recent publication of a patient survey of long COVID in younger patients described diverse symptoms with fatigue, post-exertional malaise (PEM), and cognitive dysfunction as most frequent requiring a reduced work schedule in almost half and inability to work in $22 \%$ of patients. ${ }^{3}$ PEM describes an intolerance to mental and physical exertion, which triggers an aggravation of symptoms typically lasting for more than 14 hours up to several days ${ }^{9}$.

Long-term health consequences following mild COVID-19 are poorly understood yet but have been feared based on observations from SARS-CoV-1. Here many patients were reported who developed a severe post-infectious syndrome with persistent fatigue, muscle pain, shortness 
of breath, and mental symptoms independent of illness severity. ${ }^{10}$ Various pathogens including Epstein-Barr Virus (EBV), enteroviruses, and dengue viruses are known to trigger chronic fatigue syndrome/myalgic encephalomyelitis (ME/CFS) in a subset of patients. ${ }^{11}$ It is unclear yet, if pathomechanisms of post-infectious fatigue syndromes may be different depending on the pathogen.

ME/CFS is a debilitating chronic disease with a worldwide prevalence of 0.3 to $0.8 \% .^{12}$ Profound mental and physical fatigue, sleep disturbance, and chronic pain are key symptoms of ME/CFS. The best discriminating symptoms distinguishing ME/CFS from chronic fatigue in multiple sclerosis were flu-like symptoms and the intolerance to mental and physical exertion triggering PEM for more than 14 hours. ${ }^{13} \mathrm{ME} / \mathrm{CFS}$ is classified by the World Health Organization (WHO) as neurological disease with G93.3 in the International Classification of Diseases (ICD). Although the pathomechanisms are not well understood yet, there is ample evidence of immune, autonomous nervous system and metabolic dysregulation. ${ }^{14}$ There is emerging evidence that post-infectious ME/CFS has an autoimmune mechanism. ${ }^{14-16}$

We report here on the first results of our ongoing study initiated at Charité in August 2020 to characterize patients with persistent fatigue and exertion intolerance following mild to moderate COVID-19 and to assess whether they meet diagnostic criteria for ME/CFS. Due to the complexity of symptoms patients were comprehensively evaluated by a team from various disciplines including clinical immunology, rheumatology, neurology, cardiology, endocrinology, and pulmonology with long-standing experience in diagnosing ME/CFS (https://cfc.charite.de). Our findings confirm initial concerns that COVID-19 leads to persistent fatigue syndromes in a subset of younger individuals following mild to moderate infectious disease. We describe here the clinical characteristics and biomarker findings of Chronic COVID-19 Syndrome (CCS) at six months following COVID-19 diagnosis with a subset of patients fulfilling diagnostic criteria of ME/CFS. 


\section{RESULTS}

We report here on 42 patients who presented to the Charité Fatigue Center with post COVID19 moderate to severe fatigue and exertion intolerance six months after diagnosis of COVID19. Table 1 summarizes demographic characteristics of the study population. Most patients had mild COVID-19 $(\mathrm{n}=32)$ and ten had moderate COVID-19 due to pneumonia. None of the patients required oxygen or mechanical ventilation and all but three patients were ambulatory, thus making critical illness myopathy (CIM) unlikely to explain any symptoms. Supplementary Table $\mathbf{S} 1$ shows the ten most frequent initial symptoms of COVID-19 reported by patients. 19 patients fulfilled the Canadian Consensus Criteria (CCC) for ME/CFS. These patients were referred to as Chronic COVID-19 Syndrome/CFS (CCS/CFS), the other patients as CCS. Most CCS patients (18 of 23) did not fulfill ME/CFS criteria due to a duration of PEM less than 14 hours. Further eight of 23 patients did not fulfill the criterion neurological/cognitive symptoms. Based on answers to the Patient Health Questionnaire 9 (PHQ9) we have no evidence of severe mental health issues in our study cohort (Table 1).

Table 1: Demographic and baseline clinical characteristics

\begin{tabular}{l|cc|cc|} 
& \multicolumn{2}{|c|}{ CCS/CFS (n=19) } & \multicolumn{2}{c|}{ CCS (n=23) } \\
& median & range & median & range \\
\hline age & 41 & $(24-62)$ & 36 & $(22-57)$ \\
sex (female/male) & \multicolumn{2}{|c|}{$14 / 5$} & \multicolumn{2}{c}{$15 / 8$} \\
BMI & 24,31 & $(18.1-31.8)$ & 22.49 & $(18.0-36.2)$ \\
Bell disability score & 40 & $(20-80)$ & 50 & $(30-90)$ \\
Chalder fatigue scale & 26 & $(20-31)$ & 23 & $(14-32)$ \\
PHQ9 & 12 & $(3-19)$ & 11 & $(2-18)$ \\
ESS & 9 & $(1-21)$ & 9 & $(1-17)$
\end{tabular}

CCS/CFS = chronic COVID-19 syndrome/chronic fatigue syndrome; CCS = chronic COVID-19 syndrome without fulfilling CFS criteria; $\mathrm{BMI}=$ body mass index $\left(\mathrm{kg} / \mathrm{m}^{2}\right), \mathrm{PHQ9}$ = Patient Health Questionnaire 9; ESS = Epworth Sleepiness Scale.

Two patients had an Epworth Sleepiness Scale (ESS) score of $>16$, which may indicate sleep apnea. In one patient ESS score at month three was only three when he presented already with similar fatigue severity, the other patient had an ESS score of 17. This patient was referred 
to further investigation to sleep medicine. The majority of patients were severely impaired in daily life with a median Bell disability score of 40 (range 20-80) of 100 (refers to healthy) and a SF-36 physical function score of 65 (range 15-90) of 100 (refers to healthy) in CCS/CFS and a Bell disability score of 50 (range 30-90) and SF-36 physical function score of 60 (range 2590) in CCS. Compared to CCS patients CCS/CFS patients reported lower SF-36 scores for vitality, role limitations, and social functioning ( $p=0.060,0.005,0.082$, respectively) (Fig. 1). According to the Bell disability scale patients with a score of 30-50 are able to perform light work $2-5$ hours a day $(n=28)$, thus requiring a reduced work schedule or are unable to work. Patients with a Bell disability score of $20(n=3)$ are confined to bed most of the day. ${ }^{17}$

\section{Symptom severity}

The leading symptom of fatigue was assessed by the Chalder Fatigue Questionnaire (CFQ). ${ }^{18}$ Patients classified as CCS/CFS reported a significantly higher Chalder Fatigue Score with a median of 26 (range 20-31) compared to patients with CCS with a median of 23 (range 14-32) $(p=0.006)$ (Fig. 1). The severity of PEM as the cardinal symptom of ME/CFS was the strongest discriminatory symptom. CCS/CFS patients had significantly more frequent episodes of PEM $(p=0.016)$. While according to diagnostic criteria all CCS/CFS patients had PEM of 14 hours or more, CCS patients reported PEM of less than 14 hours in 18 of 23 patients, most patients reported PEM lasting between $2-10$ hours $(n=17)$, as shown in Fig. 2. Table 2 shows the frequency and severity of symptoms at month six in patients classified as CCS/CFS and CCS. Patients with CCS/CFS reported significantly more stress intolerance $(p=0.042)$ and hypersensitivity to temperature $(p=0.024)$, noise $(p=0.029)$ and light $(p=0.014)$ (Table 2 and Suppl. Fig. S5). All other symptoms were not significantly different in frequency and severity between CCS/CFS and CCS. 
Table 2: Frequency and severity of symptoms at month 6

\begin{tabular}{|c|c|c|c|c|c|c|c|}
\hline & $\begin{array}{c}\% \\
\text { CCS/CF } \\
\text { S } \\
\text { patient } \\
\text { s }\end{array}$ & $\begin{array}{l}\text { CCS/CFS } \\
\text { media } \\
n\end{array}$ & range & $\begin{array}{c}\% \\
\text { CCS } \\
\text { patients }\end{array}$ & $\begin{array}{l}\text { media } \\
\mathrm{n}\end{array}$ & range & p-value \\
\hline Fatigue & 100 & 8 & $(5-10)$ & 100 & 7 & $(2-10)$ & 0.6874 \\
\hline PEM & 100 & 8 & $(5-10)$ & 100 & 6 & $(1-9)$ & 0.0074 \\
\hline Need for rest & 100 & 8 & $(5-10)$ & 96 & 7 & $(1-10)$ & 0.1446 \\
\hline Impaired performance in everyday life & 100 & 8 & $(4-10)$ & 96 & 8 & $(1-10)$ & 0.3454 \\
\hline Stress intolerance & 100 & 8 & $(3-10)$ & 96 & 5 & $(1-10)$ & 0.0420 \\
\hline Muscle pain & 84 & 5 & $(1-10)$ & 83 & 4 & $(1-9)$ & 0.6906 \\
\hline Headache & 95 & 5 & $(1-9)$ & 87 & 5 & $(1-10)$ & 0.4526 \\
\hline Joint pain & 89 & 3 & $(1-10)$ & 78 & 3 & $(1-9)$ & 0.4640 \\
\hline Memory/word finding problems & 100 & 5 & $(2-7)$ & 70 & 5 & $(1-8)$ & 0.6422 \\
\hline Concentration impairment & 100 & 6 & $(3-9)$ & 91 & 5 & $(1-9)$ & 0.2879 \\
\hline Mental fatigue & 100 & 6 & $(4-10)$ & 100 & 7 & $(2-10)$ & 0.6258 \\
\hline Visual disturbances & 63 & 3 & $(1-6)$ & 48 & 1 & $(1-6)$ & 0.4373 \\
\hline Palpitations & 89 & 5 & $(1-10)$ & 70 & 4 & $(1-9)$ & 0.4448 \\
\hline Dizziness when standing up & 84 & 5 & $(1-10)$ & 83 & 4 & $(1-8)$ & 0.5256 \\
\hline Dizziness when walking & 68 & 3 & $(1-7)$ & 61 & 2 & $(1-9)$ & 0.3169 \\
\hline Sleep disturbances & 89 & 7 & $(1-10)$ & 83 & 6 & $(1-10)$ & 0.9999 \\
\hline Hypersensitivity to temperature & 79 & 5 & $(1-8)$ & 48 & 1 & $(1-8)$ & 0.0241 \\
\hline Hypersensitivity to light & 84 & 5 & $(1-10)$ & 52 & 2 & $(1-7)$ & 0.0143 \\
\hline Hypersensitivity to noise & 89 & 5 & $(1-10)$ & 70 & 3 & $(1-9)$ & 0.0290 \\
\hline Breathing disorders & 79 & 5 & $(1-10)$ & 70 & 5 & $(1-10)$ & 0.6710 \\
\hline Irritable bowel & 79 & 5 & $(1-9)$ & 48 & 1 & $(1-10)$ & 0.1821 \\
\hline Fever & 21 & 1 & $(1-10)$ & 17 & 1 & $(1-3)$ & 0.8714 \\
\hline Painful lymph nodes & 32 & 1 & $(1-9)$ & 30 & 1 & $(1-7)$ & 0.9186 \\
\hline Sore throat & 63 & 3 & $(1-7)$ & 57 & 2 & $(1-7)$ & 0.4096 \\
\hline Flu-like symptoms & 79 & 5 & $(1-8)$ & 70 & 3 & $(1-10)$ & 0.0944 \\
\hline
\end{tabular}

CCS/CFS = chronic COVID-19 syndrome/chronic fatigue syndrome; CCS = chronic COVID-19 syndrome without fulfilling CFS criteria; PEM = post-exertional malaise. Symptom severity was assessed on a scale of 1-10 (none to most sever. p-values refer to symptom severity in each patient between cohorts. 


\section{Autonomic dysfunction}

The majority of patients suffered from autonomic dysfunction assessed by COMPASS 31 score with moderate (20-40) symptoms in 21 and severe (40-60) in 11 patients. ${ }^{19}$ Severity of symptoms was not significantly different between CCS/CFS and CCS. The COMPASS 31 total score and subdomains of orthostatic, gastrointestinal, vasomotor, pupillomotor, secretory, and bladder symptoms are listed in Suppl. Table S2.

\section{Hand grip strength (HGS)}

Muscle fatigue and fatigability were assessed by ten maximum hand grips at two time points (initial: Fmax1 and Fmean1 from 10 hand grips), which were repeated after 60 minutes (Fmax2 and Fmean2 from 10 hand grips). ${ }^{20}$ Compared to reference values for age-matched healthy subjects most patients in both cohorts were below the cut-off values for Fmax1/2 and Fmean1/2 discriminating healthy controls from ME/CFS. Patients with CCS/CFS had significantly lower Fmax2 and Fmean1/2 compared to CCS (Fig. 3).

\section{Sitting and standing heart rate and blood pressure}

Heart rate, systolic, and diastolic blood pressure sitting and after five minutes standing in female patients is shown in Suppl. Fig. S6. Patients with CCS/CFS had a significantly lower increase in systolic and diastolic blood pressure at standing compared to CCS. Seven patients with CFS and six with CCS had a blood pressure $>140 / 90$ sitting. Four patients with CCS/CFS and no patient with CCS fulfilled diagnostic criteria for postural tachycardia syndrome (POTS).

\section{Laboratory parameters}

Supplementary Table S3 lists the median and ranges of laboratory values, which were out of normal range in a subset of patients. Remarkably, mannose binding lectin (MBL) deficiency was more frequent with $19 \%$ in all patients compared to $5 \%$ in the general population. ${ }^{21}$ While C-reactive protein $(\mathrm{CrP})$ was slightly elevated with 7.1 and $7.6 \mathrm{mg} / \mathrm{l}$ in two patients only, IL-8 in erythrocytes indicating elevated levels during the last three to four months was above the 
normal value in $43 \%$ of all patients. ${ }^{22}$ Elevated ANA of 1:160 - 1:1280 was found in eight patients. Ds-DNA and ENA antibodies were negative in all patients and there was no evidence for a rheumatological disease. Four patients had elevated TPO antibodies with normal TSH and T3/4 values. Three of them had a diagnosis of Hashimoto thyreoiditis. The fourth patient hat only slightly elevated value $(41$, reference range $<34 \mathrm{kU} / \mathrm{l})$. Deficiencies of Vitamin $\mathrm{D}$, folic acid and ferritin were found in $17 \%, 19 \%$, and $2.5 \%$ of patients, respectively. NT-pro BNP was slightly elevated in three patients. ACE1 levels were below the normal range in $31 \%$ of patients. There were no significant differences comparing laboratory findings in CCS/CFS versus CCS. To investigate a potential pathophysiological relevance of altered laboratory parameters we correlated these laboratory values with levels of subjective fatigue assessed by questionnaires and muscular fatigue determined by HGS. Remarkably, we observed a significant inverse correlation of Fmax1/2 and Fmean1/2 with IL-8 in erythrocytes and CrP levels in the CCS and with NT-pro BNP levels in the CFS cohort (Table 3). In both cohorts we found positive correlations of HGS with levels of hemoglobin, erythrocytes, and creatinine and with ACE2 levels in the CCS and bilirubin and ferritin levels in the CFS cohort only. None of these parameters showed a correlation with fatigue severity assessed by questionnaires. However, in the CFS cohort we observed a significant inverse association of thrombocyte numbers with fatigue (1-10 scale) and strongly with the PEM score. The mean platelet volume MPV correlated inversely with thrombocytes in the CFS $(p=0.004, r=-0.627)$ but not the CCS ( $p$ $=0,725 ; r=-0,078)$ cohort indicating enhanced platelet turnover. In addition, we found an inverse association of CFQB for physical fatigue with TSH and fT3 in the CFS and of fatigue with fT4 in the CCS cohort (all $p<0.05$ ). Levels were, however, not below the normal range (Suppl. Tables S3 and S4). 
Table 3. Correlation of HGS with laboratory parameters in female patients

\begin{tabular}{|c|c|c|c|c|c|c|c|c|c|}
\hline & & & $\mathrm{CCS}$ & CFS & & & $\mathrm{CC}$ & & \\
\hline & & Fmax1 & Fmax2 & Fmean1 & Fmean2 & Fmax1 & Fmax2 & Fmean1 & Fmean2 \\
\hline $\mathrm{Hb}$ & $\mathbf{p}$ & 0.0031 & 0.0011 & 0.0002 & 0.0008 & 0.0344 & 0.0048 & 0.0070 & 0.0096 \\
\hline & $\mathbf{r}$ & 0.6412 & 0.7036 & 0.7555 & 0.7181 & 0.4634 & 0.5910 & 0.5702 & 0.5514 \\
\hline Ferritin & $\mathbf{p}$ & 0.1169 & 0.0180 & 0.0174 & 0.0175 & 0.3404 & 0.6179 & 0.4503 & 0.6359 \\
\hline & $\mathbf{r}$ & 0.3719 & 0.5501 & 0.5386 & 0.5521 & 0.2249 & 0.1188 & 0.1789 & 0.1128 \\
\hline Bilirubin & $\mathbf{p}$ & 0.0283 & 0.0764 & 0.0238 & 0.0455 & 0.9323 & 0.7671 & 0.8133 & 0.8231 \\
\hline & $\mathbf{r}$ & 0.5163 & 0.4427 & 0.5297 & 0.4942 & -0.0203 & 0.0707 & 0.0564 & 0.0534 \\
\hline Kreatinin & $\mathbf{p}$ & 0.1517 & 0.0351 & 0.0568 & 0.0465 & 0.0318 & 0.0708 & 0.0328 & 0.0749 \\
\hline & $\mathbf{r}$ & 0.3523 & 0.5178 & 0.4566 & 0.4920 & 0.4810 & 0.4123 & 0.4786 & 0.4071 \\
\hline CRP & $\mathbf{p}$ & 0.7329 & 0.6590 & 0.9649 & 0.8064 & 0.0167 & 0.0239 & 0.0514 & 0.0115 \\
\hline & $\mathbf{r}$ & -0.0839 & 0.1117 & -0.0108 & 0.0622 & -0.5160 & -0.4907 & -0.4304 & -0.5402 \\
\hline IL8 in erythrocytes & $\mathbf{p}$ & 0.3108 & 0.7789 & 0.6680 & 0.8040 & 0.0078 & 0.0365 & 0.0475 & 0.0296 \\
\hline & $\mathbf{r}$ & -0.2456 & -0.0712 & -0.1053 & -0.0630 & -0.5634 & -0.4586 & -0.4372 & -0.4748 \\
\hline NT-pro BNP & $\mathbf{p}$ & 0.0011 & 0.0001 & 0.0003 & 0.0002 & 0.4160 & 0.2484 & 0.4350 & 0.2915 \\
\hline & $\mathbf{r}$ & -0.7028 & -0.7853 & -0.7544 & -0.7626 & -0.1926 & -0.2707 & -0.1850 & -0.2481 \\
\hline ACE 1 & $\mathbf{p}$ & 0.2594 & 0.3774 & 0.3722 & 0.4097 & 0.3355 & 0.3557 & 0.5694 & 0.4858 \\
\hline & $\mathbf{r}$ & -0.2892 & -0.2279 & -0.2304 & -0.2132 & -0.2272 & -0.2180 & -0.1353 & -0.1654 \\
\hline ACE 2 & $\mathbf{p}$ & 0.5042 & 0.4856 & 0.4475 & 0.4947 & 0.1906 & 0.0224 & 0.0468 & 0.0117 \\
\hline & $\mathbf{r}$ & -0.1684 & -0.1804 & -0.1911 & -0.1767 & 0.3233 & 0.5341 & 0.4742 & 0.5795 \\
\hline
\end{tabular}

$p$ values: red $<0.05$; $r$ : blue: positive, red: negative correlations, ACE Angiotensine converting enzyme type I and II

\section{DISCUSSION}

In this study we provide evidence that COVID-19 can trigger a severe chronic syndrome with the hallmark of fatigue and exertion intolerance. Half of the patients reported here fulfill the CCC for ME/CFS. ${ }^{23}$ The others did not fulfill the CCC mostly due to shorter duration of PEM. ${ }^{9}$ Due to the overlap with ME/CFS we suggest Chronic COVID-19 Syndrome (CCS) as an appropriate terminology in accordance with another report. ${ }^{24}$ In our study, patients fulfilling the diagnostic criteria for ME/CFS had more severe fatigue and functional disability and reported more severe stress intolerance and hypersensitivity. Moreover, HGS was lower in this subgroup referred as CCS/CFS, but also considerably impaired in many of the non-CFS patients referred as CCS. Several diagnostic criteria have been proposed for use in ME/CFS, 
of which CCC are recommended for diagnosis confirmation in secondary care and in research.$^{25}$ Severity and duration of PEM is a key diagnostic criterion of the CCC. In contrast to the original minimum length of 24 hours of PEM required by the $\mathrm{CCC}$ we set the duration criterion at 14 hours, since this was shown by others to yield the highest diagnostic sensitivity and specificity to discriminate patients with ME/CFS from patients with fatigue due to other chronic illness., ${ }^{9,13}$ There are simpler diagnostic criteria including the IOM-criteria by the Institute of Medicine and the Centers for Disease Control and Prevention (CDC)-1994/Fukuda criteria but they should be used for screening purposes only as both lack key symptoms required by $\mathrm{CCC}$ for ME/CFS diagnosis. ${ }^{26,27}$ Of note, use of such less strict criteria would have classified more patients from our study as ME/CFS.

ME/CFS is a debilitating disease leading to vast social, economic, and individual impairments. ${ }^{25}$ People with ME/CFS have been struggling for decades to be recognized as having a serious and debilitating illness as many physicians are not familiar to diagnose and treat this disease. Despite the less severe phenotype of some symptoms in the CCS subgroup, most of these CCC patients were severely impaired in daily life, too. Based on the average Bell disability scores about two thirds of all patients required a reduced work schedule or were unable to work. This finding is in accordance with the recent report of a patient survey on long COVID at seven months. ${ }^{3}$

Health sequelae of long COVID-19 may be multiple. The most relevant are post-intensive care syndrome, pulmonary impairment, neurological deficits, and posttraumatic stress disorder. In our patient cohort of younger patients with mostly mild COVID-19, we have, however, no evidence for potential confounding organ impairment or major depressive or anxiety diseases in accordance with other reports. ${ }^{28,29} \mathrm{~A}$ study from a pulmonary center reported that patients with normal lung function three months after recovery from acute mild COVID-19 exhibited more fatigue and more impairment of physical functioning and quality of life than patients who had moderate-to-critical COVID-19. ${ }^{29}$ Furthermore, in this study only a minority of patients had evidence for depression or anxiety in line with our data, providing evidence that despite a high illness burden mental health is not relevantly impaired in most patients with CCS. 
There is still no specific treatment for ME/CFS and the knowledge of pathomechanisms is fragmented due to little interest and research support. ${ }^{30}$ However, there is evidence of immune, autonomic, and metabolic dysregulation in post-infectious ME/CFS. ${ }^{14}$ In line with these data, most patients in our study presented with symptoms of autonomic dysfunction. COVID-19 results in a strong inflammatory response and there is evidence for autoimmunity triggered by COVID-19. ${ }^{31}$ We have no evidence for ongoing overt inflammation as only two of the patients had mildly elevated CrP. Almost half of the patients had, however, elevated IL-8 levels in erythrocytes which are a sink for IL-8 released mainly by monocytes and endothelial cells. ${ }^{32}$ Elevated ANAs in eight patients (seven female/one male) and the preponderance of females may indicate an autoimmune mechanism similar to ME/CFS triggered by other infections. ${ }^{14-16}$ MBL deficiency has been implicated in increased susceptibility and more severe courses of infections and was found more frequently in both CCS cohorts in accordance with findings from a past study in ME/CFS. ${ }^{21}$

HGS is a reliable parameter to assess muscle fatigue and correlates with disease severity and PEM in ME/CFS. ${ }^{20}$ Remarkably, Fmax1/2 and Fmean1/2 showed a strong inverse correlation with NT-pro BNP in the CCS/CFS cohort. In the CCS cohort a significant inverse correlation of Fmax1/2 and Fmean1/2 with IL-8 in erythrocytes and CrP levels was found. Further, in both groups we found positive correlations of HGS with hemoglobin, erythrocytes and creatinine levels and with ACE2 in the CCS and bilirubin and ferritin levels in the CCS/CFS cohort only. All these biomarkers point to endothelial dysfunction and hypoperfusion as cause of muscle fatigue. A protective role on the endothelial and muscle function is provided by hemoglobulin and creatinine (oxygen or energy supply), ACE2 (vasodilation), and bilirubin (vasodilation and antioxidant). ${ }^{33}$ IL-8 and CrP levels inversely associated with HGS have a negative effect on endothelial function. Both IL-8 and IL-6 levels were significantly increased in critical compared to non-critical acute COVID-19. ${ }^{34}$ NT-pro BNP is a marker for heart failure and is released by distension of heart muscle cells, but we had no evidence for impaired cardiac function in our patients. Further BNP is produced in ischemic skeletal muscle satellite cells as a potential paracrine regulator of vasodilatation and vascular regeneration by activation of the plasmatic 
guanylate cyclase receptors. ${ }^{35}$ Thus, inverse association of NT-pro BNP levels with HGS most likely indicates endothelial dysfunction and hypoperfusion as cause of muscle fatigue, too. This finding is in accordance with recent findings in ME/CFS. ${ }^{36-38}$ Further we observed an inverse association of thrombocyte numbers with fatigue and the PEM score in the CFS cohort. Diminished thrombocytes and elevated mean platelet volume MPV indicate enhanced turnover possibly due to platelet activation. Endothelial dysfunction and endothelitis are described in the pathogenesis of acute COVID-19. ${ }^{39}$ Although our patients had normal thrombocytes this finding should prompt to study a potential role of platelet aggregates due to endothelial dysfunction and possibly ongoing endothelitis as the pathomechanism. Further we observed lower levels of TSH, fT3 and fT4 to be associated with more severe fatigue. Levels were, however, not below the normal range and may indicate thus only slightly impaired hypothalamic TRH release which requires further endocrinological evaluation. Subtle dysregulation of the hypothalamic-pituitary-adrenal (HPA) axis activity characterized by reduced adrenocorticotropic hormone $(\mathrm{ACTH})$ secretion was described in ME/CFS. ${ }^{40}$

Our study provides evidence that patients following mild COVID-19 develop a chronic syndrome fulfilling diagnostic criteria of ME/CFS in a subset. We must anticipate that this pandemic has the potential to dramatically increase numbers of ME/CFS patients. At the same time, it offers the unique chance to identify ME/CFS patients in a very early stage of disease, so that interventions such as pacing and coping can be applied early with a better therapeutic prognosis. Further, it is an unprecedented opportunity to understand the underlying pathomechanisms and characterize targets for specific treatment approaches.

\section{References}

1. Alwan, N.A. A negative COVID-19 test does not mean recovery. Nature, 170-170 (2020).

2. Williams, F.M.K., Muirhead, N. \& Pariante, C. Covid-19 and chronic fatigue. BMJ (Clinical research ed.) 370, $\mathrm{m} 2922$ (2020).

3. Davis, H.E., et al. Characterizing Long COVID in an International Cohort: 7 Months of Symptoms and Their Impact. medRxiv, 2020.2012.2024.20248802 (2020).

4. Del Rio, C., Collins, L.F. \& Malani, P. Long-term health consequences of COVID-19. Jama 324, 1723-1724 (2020). 
5. Goërtz, Y.M., et al. Persistent symptoms 3 months after a SARS-CoV-2 infection: the postCOVID-19 syndrome? ERJ open research 6(2020).

6. Ladds, E., et al. Persistent symptoms after Covid-19: qualitative study of 114 long Covid patients and draft quality criteria for services. medRxiv (2020).

7. Maxwell, E. Living with Covid 19. A dynamic review of the evidence around ongoing Covid 19 symptoms (often called Long Covid). NIHR Centre for Engagement and Dissemination (2020).

8. Logue, J.K., et al. Sequelae in Adults at 6 Months After COVID-19 Infection. JAMA Network Open 4, e210830-e210830 (2021).

9. Cotler, J., Holtzman, C., Dudun, C. \& Jason, L.A. A Brief Questionnaire to Assess PostExertional Malaise. Diagnostics (Basel) 8, 66 (2018).

10. Moldofsky, H. \& Patcai, J. Chronic widespread musculoskeletal pain, fatigue, depression and disordered sleep in chronic post-SARS syndrome; a case-controlled study. BMC neurology 11, 37 (2011).

11. Jason, L.A., et al. Predictors of post-infectious chronic fatigue syndrome in adolescents. Health Psychology and Behavioral Medicine: An Open Access Journal 2, 41-51 (2014).

12. Nacul, L.C., et al. Prevalence of myalgic encephalomyelitis/chronic fatigue syndrome (ME/CFS) in three regions of England: a repeated cross-sectional study in primary care. BMC medicine 9, 91 (2011).

13. Jason, L.A., Sunnquist, M., Brown, A. \& Reed, J. Defining Essential Features of Myalgic Encephalomyelitis and Chronic Fatigue Syndrome. Journal Of Human Behavior In The Social Environment 25, 657-674 (2015).

14. Sotzny, F., et al. Myalgic Encephalomyelitis/Chronic Fatigue Syndrome - Evidence for an autoimmune disease. Autoimmun Rev 17, 601-609 (2018).

15. Lande, A., et al. Human leukocyte antigen alleles associated with myalgic encephalomyelitis/chronic fatigue syndrome (ME/CFS). Scientific reports 10, 1-8 (2020).

16. Steiner, S., et al. Autoimmunity-Related Risk Variants in PTPN22 and CTLA4 Are Associated With ME/CFS With Infectious Onset. Front Immunol 11, 578 (2020).

17. Bell, D.S. The doctor's guide to chronic fatigue syndrome. (Addison-Wesley Publishing Co, 1995).

18. Cella, M. \& Chalder, T. Measuring fatigue in clinical and community settings. Journal of psychosomatic research 69, 17-22 (2010).

19. Sletten, D.M., Suarez, G.A., Low, P.A., Mandrekar, J. \& Singer, W. COMPASS 31: a refined and abbreviated Composite Autonomic Symptom Score. Mayo Clin Proc 87, 1196-1201 (2012).

20. Jäkel, B., et al. Hand Grip Strength and Fatigability: Correlation With Clinical Parameters and Diagnostic Suitability in ME/CFS. Journal of translational medicine accepted on Feb. 28th 2021(2021).

21. Guenther, S., et al. Frequent IgG subclass and mannose binding lectin deficiency in patients with chronic fatigue syndrome. Human immunology 76, 729-735 (2015).

22. Reinsberg, J., et al. Determination of total interleukin-8 in whole blood after cell lysis. Clin Chem 46, 1387-1394 (2000).

23. Carruthers, B.M., et al. Myalgic Encephalomyelitis/Chronic Fatigue Syndrome. Journal of Chronic Fatigue Syndrome 11, 7-115 (2003).

24. Baig, A.M. Chronic COVID Syndrome: Need for an appropriate medical terminology for LongCOVID and COVID Long-Haulers. Journal of medical virology (2020).

25. Nacul, L., et al. EUROPEAN ME NETWORK (EUROMENE) Expert Consensus on the Diagnosis, Service Provision and Care of People with ME/CFS in Europe. (2020).

26. Fukuda, K., et al. The chronic fatigue syndrome: a comprehensive approach to its definition and study. Annals of internal medicine 121, 953-959 (1994).

27. Jason, L., et al. Chronic Fatigue Syndrome vs. Systemic Exertion Intolerance Disease. Fatigue Biomed. Health Behav (2015).

28. Townsend, L., et al. Persistent fatigue following SARS-CoV-2 infection is common and independent of severity of initial infection. PloS one 15, e0240784 (2020). 
29. van den Borst, B., et al. Comprehensive health assessment three months after recovery from acute COVID-19. Clinical Infectious Diseases (2020).

30. Scheibenbogen, C., et al. The European ME/CFS Biomarker Landscape project: an initiative of the European network EUROMENE. Journal of translational medicine 15, 1-7 (2017).

31. Rodríguez, Y., et al. Autoinflammatory and autoimmune conditions at the crossroad of COVID-19. Journal of autoimmunity 114, 102506 (2020).

32. Darbonne, W.C., et al. Red blood cells are a sink for interleukin 8, a leukocyte chemotaxin. The Journal of clinical investigation 88, 1362-1369 (1991).

33. Maruhashi, T., Kihara, Y. \& Higashi, Y. Bilirubin and Endothelial Function. Journal of atherosclerosis and thrombosis 26, 688-696 (2019).

34. Zhang, X., et al. Viral and host factors related to the clinical outcome of COVID-19. Nature 583, 437-440 (2020).

35. Kuhn, M., et al. The natriuretic peptide/guanylyl cyclase--a system functions as a stressresponsive regulator of angiogenesis in mice. The Journal of clinical investigation 119, 20192030 (2009).

36. Bond, J., Nielsen, T. \& Hodges, L. Effects of Post-Exertional Malaise on Markers of Arterial Stiffness in Individuals with Myalgic Encephalomyelitis/Chronic Fatigue Syndrome. Int J Environ Res Public Health 18, 2366 (2021).

37. Scherbakov, N., et al. Peripheral endothelial dysfunction in myalgic encephalomyelitis/chronic fatigue syndrome. ESC heart failure 7, 1064-1071 (2020).

38. Wirth, K. \& Scheibenbogen, C. A Unifying Hypothesis of the Pathophysiology of Myalgic Encephalomyelitis/Chronic Fatigue Syndrome (ME/CFS): Recognitions from the finding of autoantibodies against ß2-adrenergic receptors. Autoimmun Rev 19, 102527 (2020).

39. Mosleh, W., Chen, K., Pfau, S.E. \& Vashist, A. Endotheliitis and endothelial dysfunction in patients with COVID-19: its role in thrombosis and adverse outcomes. (Multidisciplinary Digital Publishing Institute, 2020).

40. Di Giorgio, A., Hudson, M., Jerjes, W. \& Cleare, A.J. 24-hour pituitary and adrenal hormone profiles in chronic fatigue syndrome. Psychosomatic medicine 67, 433-440 (2005).

41. Bell, D. The Doctor's Guide To Chronic Fatigue Syndrome: Understanding. (Treating, And Living With Cfids: Da Capo Press, 1995).

42. Agarwal, A.K., Garg, R., Ritch, A. \& Sarkar, P. Postural orthostatic tachycardia syndrome. Postgraduate medical journal 83, 478-480 (2007).

\section{METHODS}

\section{Study protocol}

The primary objective of this monocentric prospective observational study is to characterize patients contacting the Charité Fatigue Center with persistent fatigue and exertion intolerance after COVID-19 and determine if they fulfill diagnostic criteria for ME/CFS. Patients were selected based on a screening questionnaire, specifying COVID-19 diagnosis and symptoms including mild to moderate COVID-19 according to WHO criteria and persistent symptoms of moderate to severe fatigue and exertion intolerance six months post infection in the absence of relevant cardiac, respiratory, neurological, or psychiatric comorbidity. A subset of patients was already seen at months three and four, and reevaluated at month six. COVID-19 had been diagnosed by PCR (polymerase chain reaction) or 
serology (SARS-CoV $2 \lg G / \lg A$ ). Three patients with no PCR and negative serology were included due to typical initial symptoms including loss of smell and taste. All patients signed informed consent before study assessment. This study is part of the Pa-COVID-19 study of Charité and approved by the Ethics Committee of Charité Universittsmedizin Berlin in accordance with the 1964 Declaration of Helsinki and its later amendments (EA2/066/20). Between August and November 2020 a total of 57 patients presented at our outpatient clinics. Patients were excluded from this study in case of relevant comorbidities or preexisting fatigue, absence of confirmed COVID-19 or evidence of organ dysfunction. Furthermore, patients were not included in this report in case of pending cardiopulmonary or neurological assessment. Thus, we report here on the results of the cross-sectional analysis at month six in a total of 42 patients.

\section{Diagnostic criteria and symptom assessment}

Severity of mental and physical fatigue was assessed using the Chalder Fatigue Score (CFQ). The first seven questions assess mental fatigue (CFQA), the last four physical fatigue (CFQB). Disability and daily physical function were assessed by the Bell disability scale and Short Form Health Survey-36 (SF36 Version 1). The Bell disability scale is scored from 0 (very severe, bedridden constantly) to 100 (healthy). ${ }^{41}$ Frequency and the severity of PEM symptoms were assessed according to Cotler et al. ${ }^{9}$. Symptoms of autonomic dysfunction were assessed by the Composite Autonomic Symptom Score (COMPASS 31). ${ }^{19}$ Depression and sleepiness were assessed by Patient Health Questionnaire 9 (PHQ9) and Epworth Sleepiness Scale (ESS). According to PHQ9 patients were classified as having minimal depressive symptoms (1-4), mild depressive symptoms (5-9), moderate depressive symptoms (10-14), moderately severe depressive symptoms (15-19), or severe depressive symptoms (20-27). According to ESS patients were classified as no evidence of sleep apnea (0-9), possible mild to moderate sleep apnea (11-15), or possible severe sleep apnea (>16). One patient with a PHQ9 score of $>20$ who is in ongoing psychosomatic evaluation was excluded from this report.

A diagnosis of ME/CFS was based on Canadian Consensus criteria (CCC) and exclusion of other diseases, which may explain chronic fatigue and potential confounding comorbidities. ${ }^{23}$ In contrast to the original classification and in accordance with the studies of $L$. Jason and colleagues a minimum of 14 hours of PEM instead of 24 hours was required for diagnosis of ME/CFS. ${ }^{9}$ Key symptoms of CCC were quantified using a 1-10 scale. All data was recorded using a REDCap database. 


\section{Functional studies, imaging and laboratory values}

The hand grip strength (HGS) was assessed using an electric dynamometer assessing maximal and mean force of ten times repeated maximal pulls (Fmax1 and Fmean1) and a second assessment 60 minutes later (Fmax2 and Fmean2). ${ }^{20}$ Blood pressure and heart rate were assessed sitting, at time point zero, after two, five, and ten minutes standing. Postural tachycardia syndrome (POTS) is defined as increase of $>30 \mathrm{bpm}$ or over $120 \mathrm{bpm}$ within ten minutes standing and orthostatic intolerance. ${ }^{42}$ Most patients had already had neurological, pulmonary, and cardiac assessment before referral to our outpatient clinic without evidence for relevant impairment or comorbidity. In patients who reported moderate to severe difficulties with breathing, chest computer tomography (CT) and pulmonary function tests were performed. Patients who reported severe cognitive impairment or severe headache got a further comprehensive neurological assessment from our neurology team. Patients with sitting or postural tachycardia or elevated NT-pro BNP got a cardiologic examination including ECG, 24h ECG and echocardiography from our cardiology team. Laboratory parameters including Full blood count and differential $(\mathrm{CBC})$, lymphoycte subsets, IL-8 in erythrocytes, mannose binding lectin (MBL), CrP, immunoglobulins, ANA, ENA, C3/4, anti-TPO antibodies, TSH, fT3/4, ferritin, creatinine, liver enzymes, ACE1/2, NT-pro BNP were determined at the Charité diagnostics laboratory (Labor Berlin GmbH, Berlin, Germany). ACE2 was assessed by an ELISA (R\&D Systems).

\section{Patient and Public Involvement}

The German facebook group of patients with long COVID contacted us first in August 2020 sharing their observations (https://c19langzeitbeschwerden.de/). The study design was developed based on frequency, type and severity of symptoms reported and discussed with the patient group. The possibility for local patients to participate in our study was communicated on their website.

\section{Statistical analysis}

We performed the statistical analysis with Excel GraphPad Prism 6.0. We used the non-parametric Mann-Whitney test to analyze differences between groups. For comparison of pulse and blood pressure at different time points we used the Wilcoxon test. For correlation analyses we calculated non-parametric Spearman correlation coefficients. A p-value of $<0.05$ was considered as statistically significant. Due to multiple testing $p$-values are considered descriptive without adjustments for multiple comparisons. 
Author contributions: CSc, CK, and FP developed the concept of the study. HDV gave important input into study concept and objectives. HF and CK were responsible for data curation and analyses of data. CK, CSc, KW, LH, FS, JBS, LMA, FP, BH, CSk, JS, and TB were involved in clinical investigation. MH and RG were involved in data transfer and patient care. TZ was involved in ethical affairs and data management. HF, CK, and CSc validated the data. HF was involved in data visualization. CSc wrote the original draft of the manuscript. $\mathrm{CK}, \mathrm{HF}$, and UB reviewed and edited the manuscript. All authors revised and approved the manuscript. The corresponding author attests that all listed authors meet authorship criteria and that no others meeting the criteria have been omitted. CSc is the guarantor.

Funding: This work is supported by a grant from the Weidenhammer-Zöbele Foundation.

Competing interests: $\mathrm{HH}$ is the owner of CellTrend. All other authors declare that they have no conflict of interests.

Ethical approval: Ethical approval was given by the Ethics Committee of Charité Universitätsmedizin Berlin in accordance with the 1964 Declaration of Helsinki and its later amendments (EA2/066/20).

Data sharing: Pseudonomized patient data may be available upon written request to the corresponding author. Additional documents, including informed consent form are available as supplementary files within the original submission.

Informed consent: All participants provided written informed consent.

Transparency: The lead author (the manuscript's guarantor) affirms that the manuscript is an honest, accurate, and transparent; that no important aspects of the study have been omitted; and that any discrepancies from the study as planned have been explained. Dissemination to participants and related patient and public communities is encouraged by open access publication and citing the study on our site https://cfc.charite.de/. We are engaging with print and internet press, television, radio, news, and documentary program makers.

Acknowledgements: We thank Silvia Thiel for patient care and data management. We thank all patients who gave us their consent to publish their data in this study. 


\section{List of Figures:}

Fig. 1 Severity of fatigue and disability

Fig. 2 Frequency, severity, and length of post-exertional malaise (PEM)

Fig. 3 Hand grip strength (HGS) 
Bell

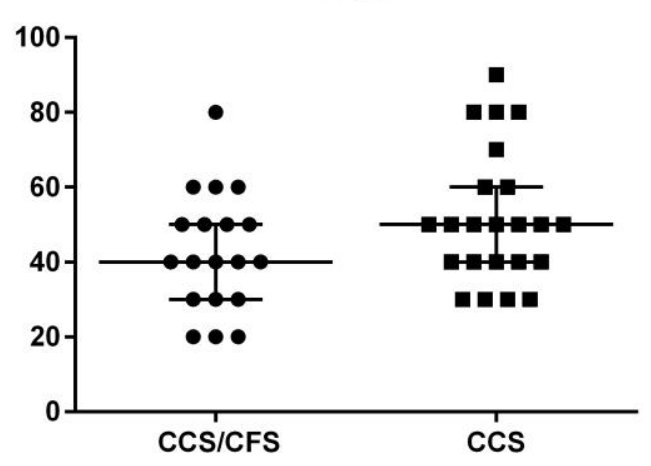

SF-36 physical functioning

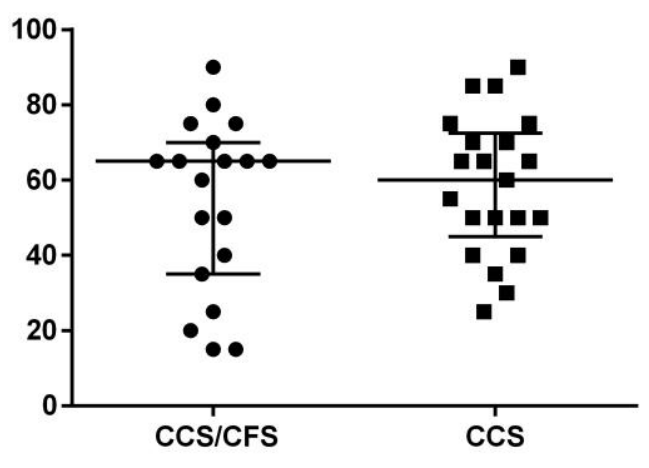

SF-36 role limitations due to physical health

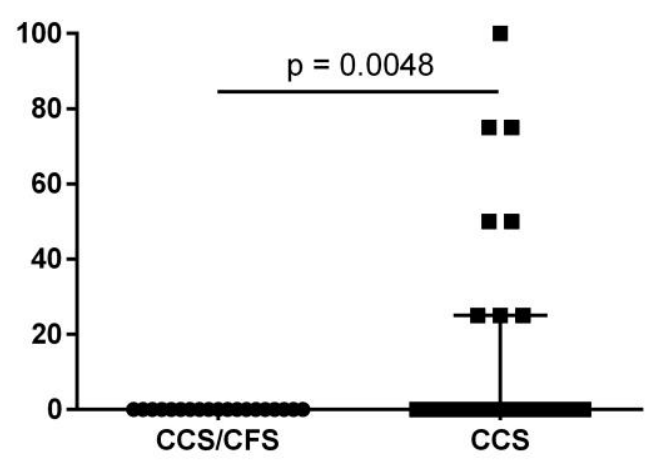

Chalder

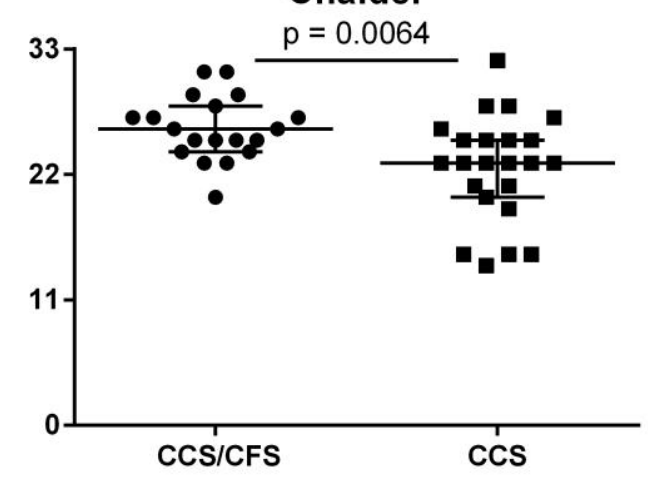

SF-36 vitality

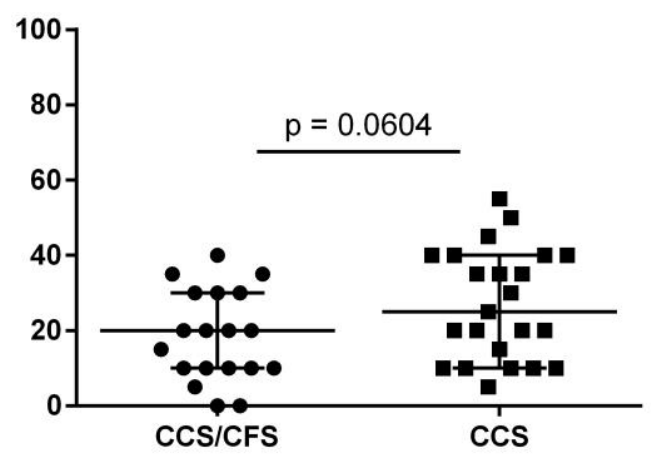

SF-36 social functioning

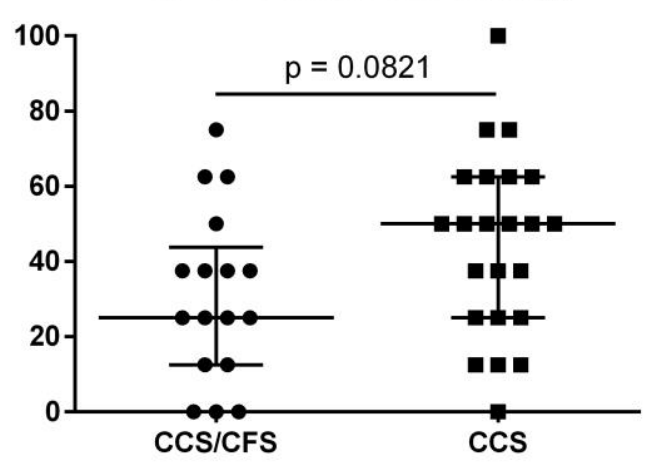

Fig. 1 Severity of fatigue and disability

Bell disability score (score 0-100), Chalder fatigue score (score 0-33), and SF-36 physical function, vitality, role limitations, and social function (scores $0-100$ ) in 19 CCS/CFS (17 for social functioning) and $23 \operatorname{CCS}$ ( 21 for physical functioning) patients. Median, range, and single values are shown. 


\section{PEM frequency}

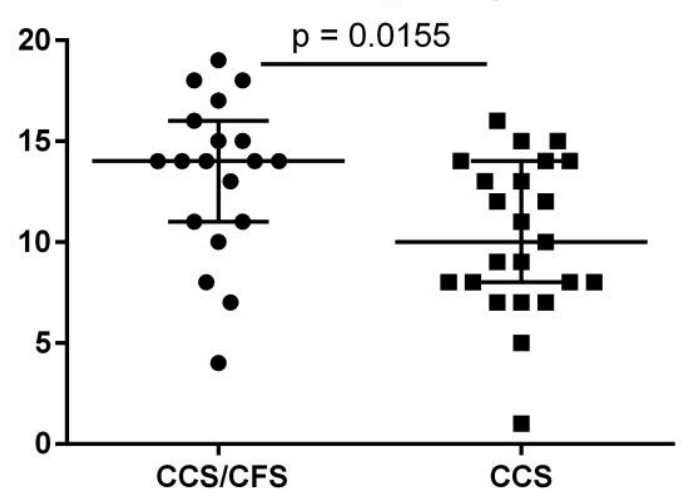

PEM severity

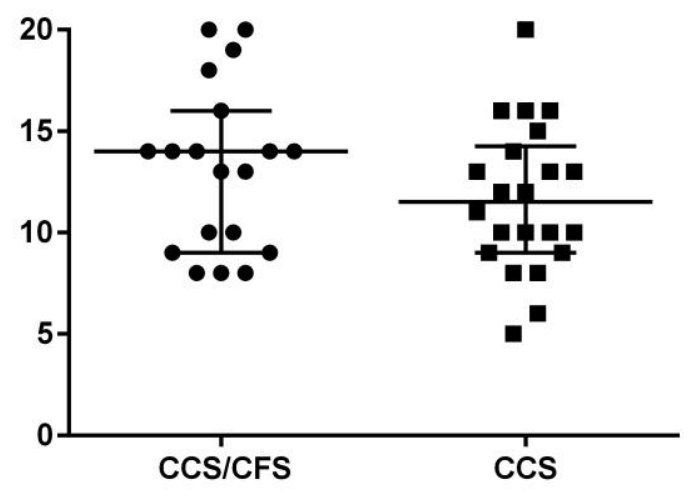

PEM length

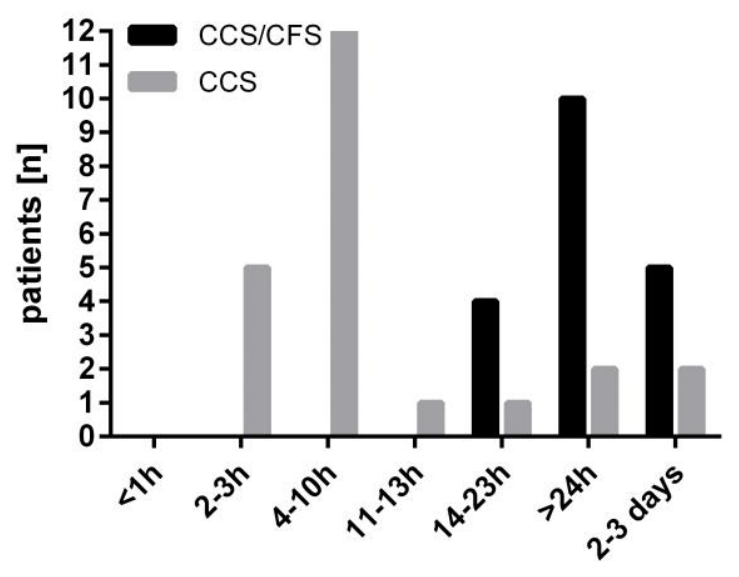

Fig. 2 Frequency, severity and length of post exertional malaise (PEM)

Frequency and severity of PEM was assessed on a five items scale with $0-20$ points (none to severest) and the length in seven categories (from $<1 \mathrm{~h}$ to $2-3$ days) according to Cotler. ${ }^{9}$ Median, range, and single values are shown in 19 CCS/CFS and 23/22 CCS patients (one patient completed only frequency block). 
Fmax1

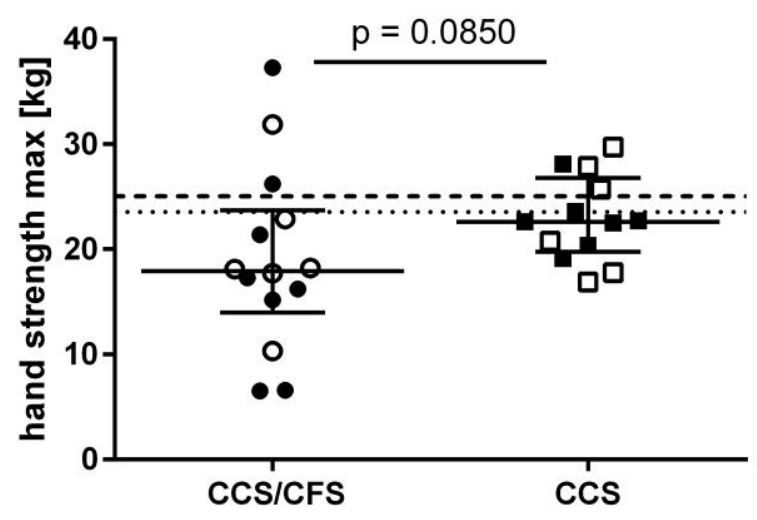

Fmean1

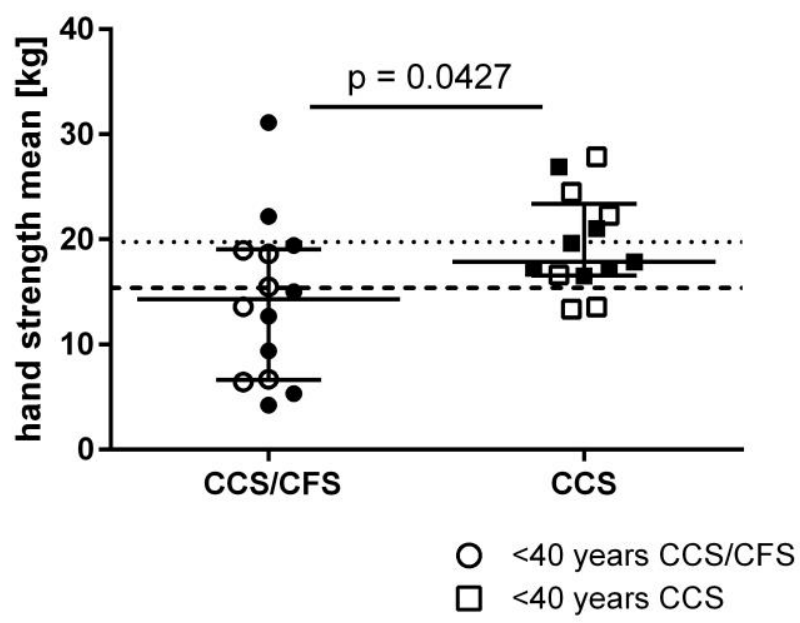

Fmax2

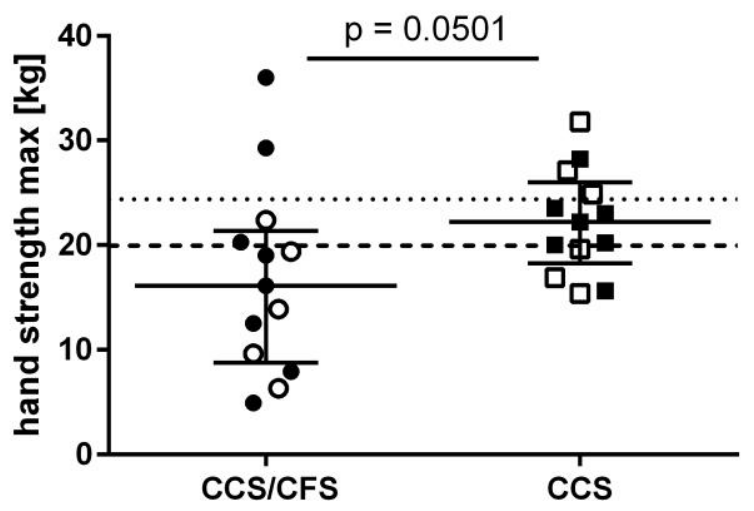

\section{Fmean2}

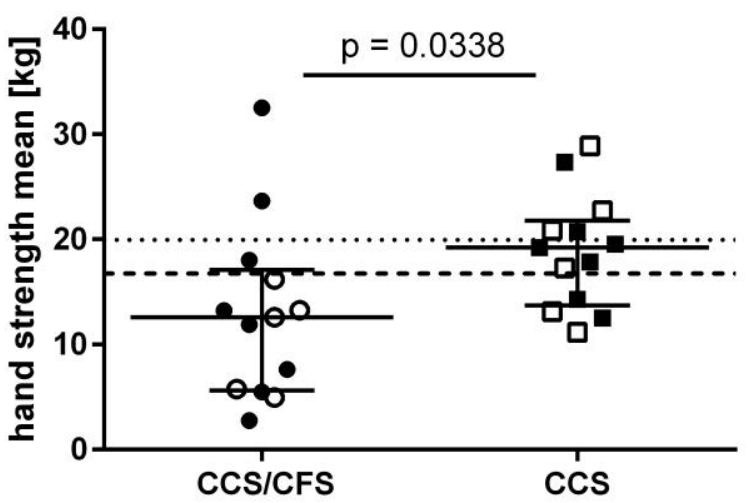

... Cut-off $<40$ years

- - Cut-off $>40$ years

Fig. 3 Hand grip strength (HGS)

Fmax1 and Fmean 1 of ten pulls in 14 female patients and repeat assessment after 60 minutes (Fmax2 and Fmean2 of ten pulls) in 13 female CCS/CFS and 13 female CCS patients are shown (median, range, and single values). Cut-off values of AUC reference values for age-matched healthy females are shown as dashed lines $(<40$ years black dots and narrower dashed lines. $>40$ years white dots and wider dashed lines). 


\section{Supplemental material}

Table S1: Ten most frequent initial symptoms of COVID-19 reported by patients

\begin{tabular}{l|cccc|cccc|cc|} 
& \multicolumn{5}{|c|}{ CCS/CFS } & \multicolumn{4}{c|}{ cCS } & \multicolumn{2}{c|}{ all patients } \\
& male & female & total & \% CCS/CFS & male & female & total & \% CCS & total & \% total \\
\hline $\mathrm{n}$ & 5 & 14 & 19 & & 8 & 15 & 23 & & 42 & \\
\hline Fatigue/malaise & 4 & 9 & $\mathbf{1 3}$ & 68 & 7 & 15 & $\mathbf{2 2}$ & 96 & $\mathbf{3 5}$ & 83 \\
Fever/ high temperature & 3 & 9 & $\mathbf{1 2}$ & 63 & 6 & 10 & $\mathbf{1 6}$ & 70 & $\mathbf{2 8}$ & 67 \\
\hline Cough & 2 & 8 & $\mathbf{1 0}$ & 53 & 7 & 8 & $\mathbf{1 5}$ & 65 & $\mathbf{2 5}$ & 60 \\
Resp. problems & 2 & 8 & $\mathbf{1 0}$ & 53 & 5 & 9 & $\mathbf{1 4}$ & 61 & $\mathbf{2 4}$ & 57 \\
\hline Loss of smell & 0 & 10 & $\mathbf{1 0}$ & 53 & 5 & 8 & $\mathbf{1 3}$ & 57 & $\mathbf{2 3}$ & 55 \\
\hline Loss of taste & 0 & 10 & $\mathbf{1 0}$ & 53 & 4 & 8 & $\mathbf{1 2}$ & 52 & $\mathbf{2 2}$ & 52 \\
\hline Headache & 2 & 9 & $\mathbf{1 1}$ & 58 & 2 & 9 & $\mathbf{1 1}$ & 48 & $\mathbf{2 2}$ & 52 \\
Arthralgia & 0 & 8 & $\mathbf{8}$ & 42 & 2 & 7 & $\mathbf{9}$ & 39 & $\mathbf{1 7}$ & 40 \\
Chest pain & $\mathbf{2}$ & 6 & $\mathbf{8}$ & 42 & 3 & 6 & $\mathbf{9}$ & 39 & $\mathbf{1 7}$ & 40 \\
\hline Gastrointestinal symptoms & 1 & 8 & $\mathbf{9}$ & 47 & 1 & 6 & $\mathbf{7}$ & 30 & $\mathbf{1 6}$ & 38
\end{tabular}

Table S2: COMPASS 31 total score and subdomains

\begin{tabular}{|l|c|ccc|ccc|}
\hline COMPASS 31 & & \multicolumn{3}{|c|}{ CCS/CFS } & \multicolumn{3}{c|}{ CCS } \\
& max. value & \% & median & range & \% & median & range \\
\hline total & 100 & 100 & $\mathbf{3 9 . 3 7}$ & $(7.1-62.2)$ & 100 & $\mathbf{2 6 . 7 9}$ & $(2.5-54.0)$ \\
\hline Orthostasis & 40 & 84 & $\mathbf{2 4 . 0 0}$ & $(0-40)$ & 76 & $\mathbf{1 6 . 0 0}$ & $(0-40)$ \\
Vasomotor & 5 & 26 & $\mathbf{0 . 0 0}$ & $(0-4.2)$ & 10 & $\mathbf{0 . 0 0}$ & $(0-4.2)$ \\
Secretomotor & 15 & 63 & $\mathbf{4 . 2 9}$ & $(0-12.9)$ & 57 & $\mathbf{2 . 1 4}$ & $(0-10.7)$ \\
Gastrointestinal & 25 & 89 & $\mathbf{6 . 2 5}$ & $(0-15.2)$ & 95 & $\mathbf{6 . 2 5}$ & $(0-16.1)$ \\
Bladder & 10 & 47 & $\mathbf{0 . 0 0}$ & $(0-3.3)$ & 33 & $\mathbf{0 . 0 0}$ & $(0-2.2)$ \\
Pupillomotor & 5 & 95 & $\mathbf{1 . 6 7}$ & $(0-3.3)$ & 86 & $\mathbf{1 . 3 3}$ & $(0-3.0)$ \\
\hline
\end{tabular}

CCS/CFS = chronic COVID-19 syndrome/chronic fatigue syndrome; CCS = chronic COVID-19 syndrome without fulfilling CFS criteria; COMPASS 31 = Composite Autonomic Symptom Score-31 
Table S3 Laboratory values

\begin{tabular}{|c|c|c|c|c|c|c|c|c|c|c|c|c|c|c|c|}
\hline & \multirow[b]{2}{*}{ unit } & \multicolumn{6}{|c|}{ CCS/CFS } & \multicolumn{6}{|c|}{ CCS } & \multicolumn{2}{|c|}{ Reference range } \\
\hline & & $n$ & median & $\min$ & $\max$ & $\%$ low & $\%$ high & $\mathrm{n}$ & median & $\min$ & $\max$ & $\%$ low & $\%$ high & $\mathbf{m}$ & f \\
\hline CD4 Tcells abs & $/ \mathrm{nl}$ & 19 & 0.75 & 0.31 & 2.07 & 5 & 11 & 23 & 0.72 & 0.31 & 1.52 & 17 & 4 & \multicolumn{2}{|c|}{$0.5-1.2$} \\
\hline CD8 Tcells abs & $/ \mathrm{nl}$ & 19 & 0.41 & 0.21 & 0.97 & 16 & 11 & 23 & 0.37 & 0.21 & 0.61 & 35 & 0 & \multicolumn{2}{|c|}{$0.3-0.8$} \\
\hline Erythroctes & $/ p l$ & 19 & 4.5 & 4 & 5 & 0 & 0 & 23 & 4.7 & 4 & 5.4 & 0 & 0 & $4.3-5.8$ & $3.9-5.2$ \\
\hline $\mathrm{Hb}$ & $\mathrm{g} / \mathrm{dl}$ & 19 & 13.7 & 12.2 & 15.4 & 0 & 0 & 23 & 13.7 & 12.2 & 17.6 & 0 & 4 & $13.5-17.0$ & $12.0-15.6$ \\
\hline Thtomboyctes & $/ \mathrm{nl}$ & 19 & 263 & 143 & 376 & 5 & 5 & 23 & 233 & 168 & 452 & 0 & 4 & \multicolumn{2}{|c|}{$150-370$} \\
\hline Ferritine & $\mu \mathrm{g} / \mathrm{l}$ & 19 & 91.9 & 16.7 & 337.2 & 0 & 16 & 21 & 69.9 & 11.2 & 235.1 & 5 & 5 & $30-400$ & $13-150$ \\
\hline Creatinine & $\mathrm{mg} / \mathrm{dl}$ & 18 & 0.785 & 0.61 & 1.04 & 0 & 22 & 21 & 0.77 & 0.61 & 0.99 & 5 & 5 & $0.7-1.2$ & $0.5-0.9$ \\
\hline Creatinkinase & $\mathrm{U} / \mathrm{I}$ & 19 & 74 & 25 & 152 & - & 0 & 21 & 61 & 41 & 273 & - & 5 & $<190$ & $<167$ \\
\hline $\mathrm{LDH}$ & $\mathrm{U} / \mathrm{I}$ & 19 & 205 & 147 & 317 & 0 & 16 & 22 & 195 & 124 & 280 & 5 & 5 & \multicolumn{2}{|c|}{$135-250$} \\
\hline Bilirubin & $\mathrm{mg} / \mathrm{dl}$ & 18 & 0.505 & 0.22 & 1.6 & - & 6 & 22 & 0.49 & 0.2 & 0.97 & - & 0 & \multicolumn{2}{|c|}{$<1.2$} \\
\hline GPT & $\mathrm{U} / \mathrm{I}$ & 18 & 17.5 & 11 & 49 & - & 11 & 21 & 18 & 10 & 49 & - & 10 & $<41$ & $<31$ \\
\hline GOT & $\mathrm{U} / \mathrm{I}$ & 18 & 24.5 & 17 & 33 & - & 0 & 21 & 22 & 15 & 37 & - & 0 & $<49$ & $<35$ \\
\hline NT-pro BNP & $\mathrm{ng} / \mathrm{l}$ & 18 & 47 & 7 & 125 & - & 11 & 21 & 39 & 6 & 181 & - & 5 & $<121$ & $<97$ \\
\hline ACE 1 & $\mathrm{U} / \mathrm{I}$ & 17 & 26.8 & 10.3 & 52.5 & 29 & 0 & 22 & 24.85 & 12.2 & 37.6 & 32 & 0 & \multicolumn{2}{|c|}{$20-70$} \\
\hline ACE 2 & $\mathrm{ng} / \mathrm{ml}$ & 18 & 4.05 & 2.5 & 541.7 & 6 & 17 & 20 & 4.6 & 2.7 & 387.6 & 0 & 25 & \multicolumn{2}{|c|}{ 2.6-7. } \\
\hline $\mathrm{fT} 3$ & $\mathrm{ng} / \mathrm{l}$ & 18 & 3.325 & 2.54 & 3.98 & 0 & 0 & 23 & 3.27 & 2.57 & 4.08 & 0 & 0 & \multicolumn{2}{|c|}{$2-4.4$} \\
\hline fT4 & $\mathrm{ng} / \mathrm{l}$ & 18 & 13.8 & 11 & 18.1 & 0 & 11 & 23 & 13.1 & 9.77 & 15.9 & 0 & 0 & \multicolumn{2}{|c|}{$9.3-17$} \\
\hline TSH basal & $\mathrm{mU} / \mathrm{l}$ & 18 & 1.385 & 0.37 & 2.36 & 0 & 0 & 21 & 1.33 & 0.7 & 3.31 & 0 & 0 & \multicolumn{2}{|c|}{$0.27-4.2$} \\
\hline Anti-TPO Ab & $\mathrm{kU} / \mathrm{l}$ & 15 & 9 & 9 & 328 & - & 13 & 19 & 9 & 9 & 81 & - & 11 & \multicolumn{2}{|c|}{$<34$} \\
\hline $\lg G$ & $\mathrm{~g} / \mathrm{l}$ & 19 & 10.94 & 7.13 & 13.22 & 0 & 0 & 23 & 10.63 & 6.73 & 16.18 & 4 & 4 & \multicolumn{2}{|c|}{$7-4$} \\
\hline $\lg A$ & $g / l$ & 19 & 1.75 & 1.01 & 4.09 & 0 & 11 & 23 & 1.92 & 0.32 & 4.62 & 4 & 4 & \multicolumn{2}{|c|}{$0.7-4$} \\
\hline $\operatorname{IgM}$ & $\mathrm{g} / \mathrm{l}$ & 19 & 1.15 & 0.63 & 5.05 & 0 & 11 & 23 & 1.09 & 0.45 & 13.48 & 0 & 9 & \multicolumn{2}{|c|}{$0.4-2.3$} \\
\hline $\lg \mathrm{E}$ & $\mathrm{g} / \mathrm{l}$ & 19 & 46 & 2.4 & 1072 & 0 & 21 & 23 & 35.1 & 4 & 751 & 0 & 22 & \multicolumn{2}{|c|}{$0-100$} \\
\hline $\operatorname{lgG1}$ & $\mathrm{g} / \mathrm{l}$ & 19 & 5.797 & 3.637 & 7.226 & 0 & 0 & 22 & 6.034 & 3.13 & 8.209 & 0 & 5 & \multicolumn{2}{|c|}{$2.8-8$} \\
\hline $\lg G 2$ & $\mathrm{~g} / \mathrm{l}$ & 19 & 3.904 & 2.607 & 6.445 & 0 & 5 & 22 & 4.881 & 2.752 & 5.934 & 0 & 5 & \multicolumn{2}{|c|}{$1.12-5.7$} \\
\hline $\lg G 3$ & $\mathrm{~g} / \mathrm{l}$ & 19 & 0.473 & 0.15 & 1.342 & 5 & 5 & 22 & 0.505 & 0.19 & 1.062 & 5 & 0 & \multicolumn{2}{|c|}{$0.24-1.25$} \\
\hline $\lg \mathrm{g} 4$ & $\mathrm{~g} / \mathrm{l}$ & 19 & 0.262 & 0.132 & 2.115 & 0 & 5 & 22 & 0.544 & 0.096 & 1.781 & 0 & 9 & \multicolumn{2}{|c|}{$0.052-1.25$} \\
\hline C3 & $\mathrm{mg} / \mathrm{l}$ & 18 & 1080 & 850 & 1640 & 11 & 0 & 22 & 1155 & 820 & 1720 & 14 & 0 & & 1800 \\
\hline $\mathrm{C} 4$ & $\mathrm{mg} / \mathrm{l}$ & 18 & 185 & 100 & 330 & 0 & 0 & 22 & 210 & 110 & 320 & 0 & 0 & & 400 \\
\hline $\mathrm{MBL}$ & $\mathrm{ng} / \mathrm{ml}$ & 18 & 1452 & $<50$ & 4000 & 17 & - & 22 & 3734 & $<50$ & 4000 & 23 & - & & \\
\hline IL8 in erythroc. & $\mathrm{pg} / \mathrm{ml}$ & 19 & 134.8 & 97 & 224 & - & 37 & 23 & 149.2 & 65.2 & 442 & - & 48 & & \\
\hline Soluble IL2R & $\mathrm{IU} / \mathrm{ml}$ & 19 & 312 & 176 & 746 & - & 5 & 23 & 326 & 173 & 765 & - & 4 & & \\
\hline CRP & $\mathrm{mg} / \mathrm{l}$ & 19 & 0.8 & 0.6 & 7.1 & - & 5 & 23 & 1 & 0.6 & 7.57 & - & 4 & & \\
\hline Vitamin D3 & $\mathrm{nmol} / \mathrm{l}$ & 19 & 80.4 & 46.2 & 109.6 & 11 & 0 & 23 & 62.4 & 16.7 & 217.6 & 22 & 4 & & \\
\hline Folic acid & $\mu \mathrm{g} / \mathrm{l}$ & 16 & 7.3 & 2.5 & 20 & 19 & 13 & 21 & 9.7 & 2.7 & 20 & 19 & 5 & & 18.7 \\
\hline
\end{tabular}

abs: absolute; R: receptor; NT-pro BNP reference values according to age: $\leq 44$ years $=\leq 97, \leq 54$ years $=\leq 121, \leq 64$ years $=\leq 198, \leq 74$ years $=\leq 285,<74$ years $=\leq 526$ 
Table S4 Correlation of TSH, T3 and T4 and thrombocytes with fatigue and PEM by CFQ and 1-10 Likert scale.

\begin{tabular}{|c|c|c|c|c|c|c|c|c|c|c|c|}
\hline & \multicolumn{5}{|c|}{ CCS/CFS } & \multicolumn{5}{|c|}{$\operatorname{ccs}$} \\
\hline & & CFQ & CFQA & CFQB & Fatigue (1-10) & PEM & CFQ & CFQA & CFQB & Fatigue $(1-10)$ & PEM \\
\hline \multirow[t]{2}{*}{ fT3 } & $\mathbf{p}$ & 0.0114 & 0.6720 & 0.0307 & 0.5472 & 0.4820 & 0.8374 & 0.7023 & 0.1863 & 0.5928 & 0.4114 \\
\hline & $r$ & -0.5813 & -0.1072 & -0.5098 & -0.1520 & 0.1771 & 0.0453 & -0.0843 & 0.2857 & -0.1177 & 0.1799 \\
\hline \multirow[t]{2}{*}{ fT4 } & $\mathbf{p}$ & 0.7020 & 0.8116 & 0.3646 & 0.4409 & 0.0729 & 0.2849 & 0.4934 & 0.2152 & 0.0296 & 0.9875 \\
\hline & $\mathbf{r}$ & -0.0969 & 0.0605 & -0.2272 & -0.1938 & -0.4326 & -0.2329 & -0.1504 & -0.2686 & -0.4538 & 0.0035 \\
\hline \multirow[t]{2}{*}{ TSH } & $\mathbf{p}$ & 0.0844 & 0.9786 & 0.0209 & 0.9060 & 0.3799 & 0.4276 & 0.8678 & 0.0973 & 0.4339 & 0.7812 \\
\hline & $\mathbf{r}$ & -0.4179 & 0.0068 & -0.5393 & 0.0300 & 0.2202 & 0.1828 & 0.0387 & 0.3714 & 0.1804 & 0.0645 \\
\hline \multirow[t]{2}{*}{ Thrombocytes } & $\mathbf{p}$ & 0.7115 & 0.1198 & 0.6177 & 0.0012 & 0.0002 & 0.8703 & 0.9874 & 0.9504 & 0.4875 & 0.1847 \\
\hline & $\mathbf{r}$ & -0.0908 & -0.3692 & 0.1224 & -0.6846 & -0.7488 & -0.0360 & -0.0035 & 0.0137 & 0.1524 & -0.2867 \\
\hline
\end{tabular}

Severity of mental and physical fatigue was assessed using a 1 - 10 Likert scale and the Chalder Fatigue Score (CFQ). The first seven questions assess mental fatigue (CFQA), the last four physical fatigue (CFQB). PEM score is the sum of frequency and severity of PEM with $0-20$ points each and the length with 6 points according to Cotler.(9)
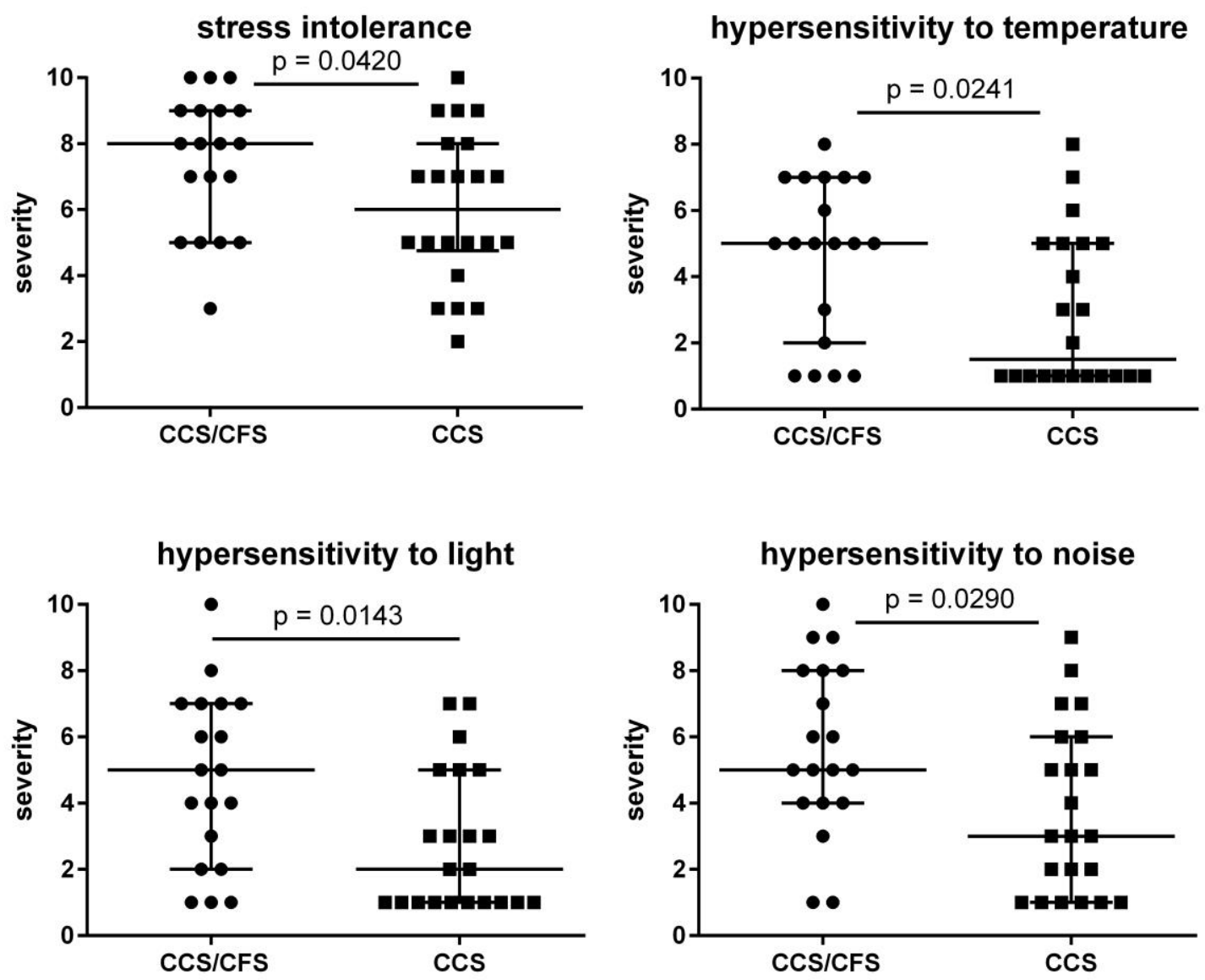

Fig. S5 Severity of stress intolerance and of hypersensitivity to temperature, light, and noise 
Severity of symptoms was assessed on a $1-10$ Likert scale (none to severest). Median, range and single values are shown in 19 CCS/CFS and 22 CCS patients.
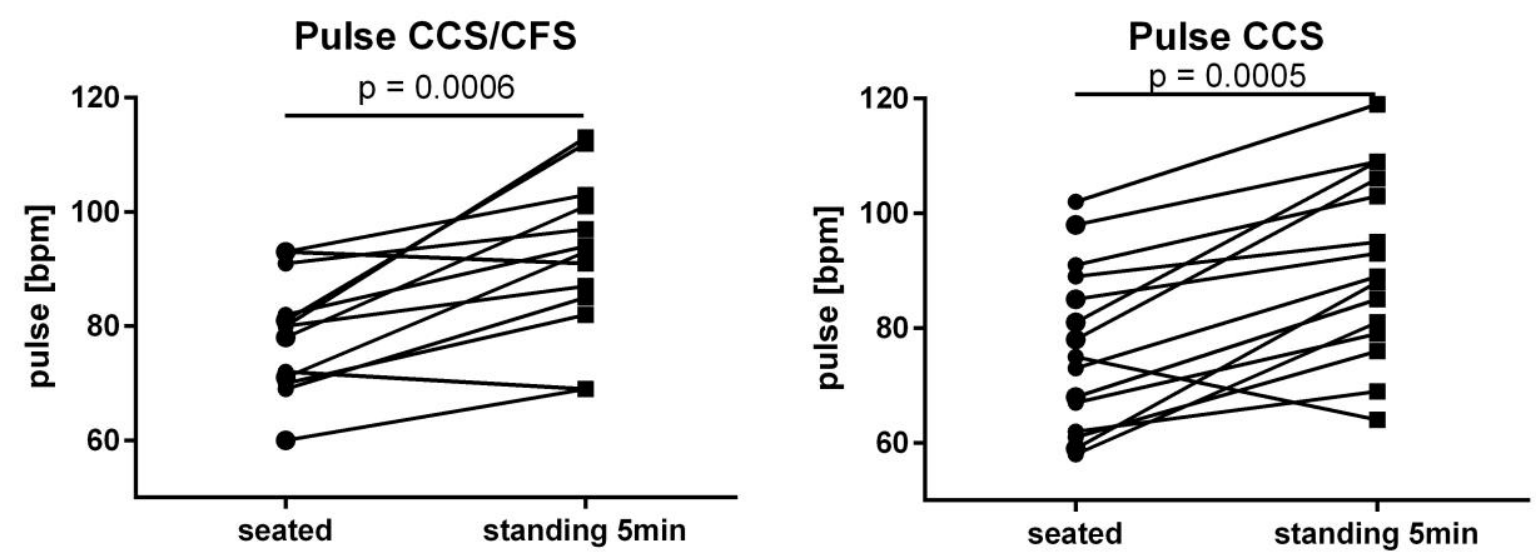

\section{Systole CCS/CFS}

\section{Systole CCS}
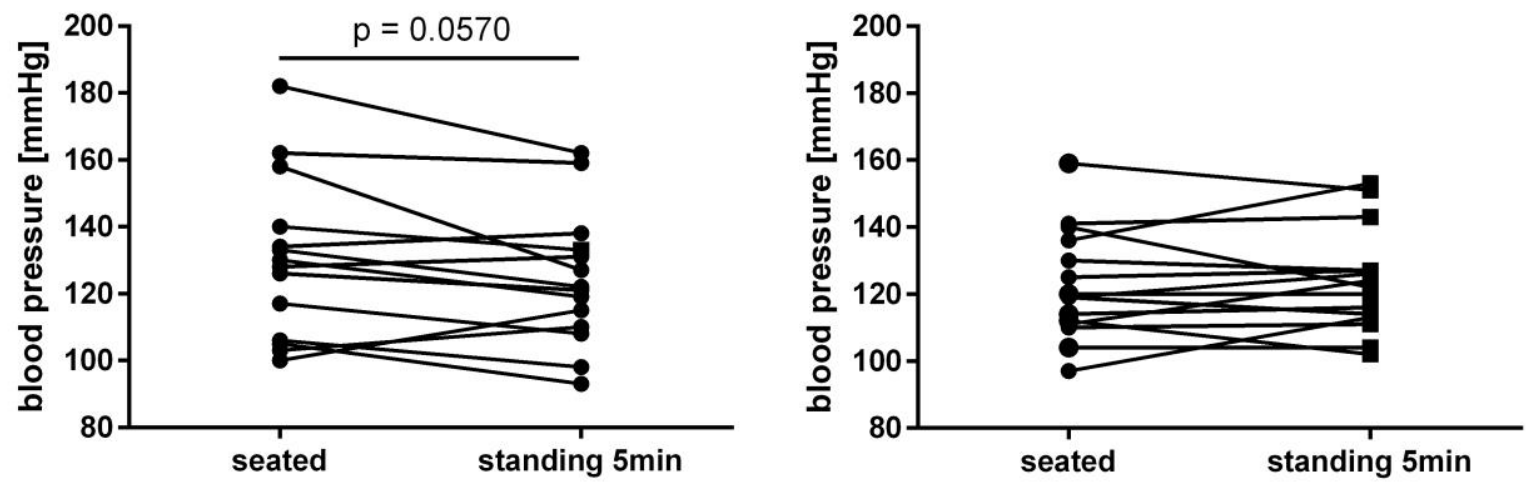

\section{Diastole CCS/CFS}
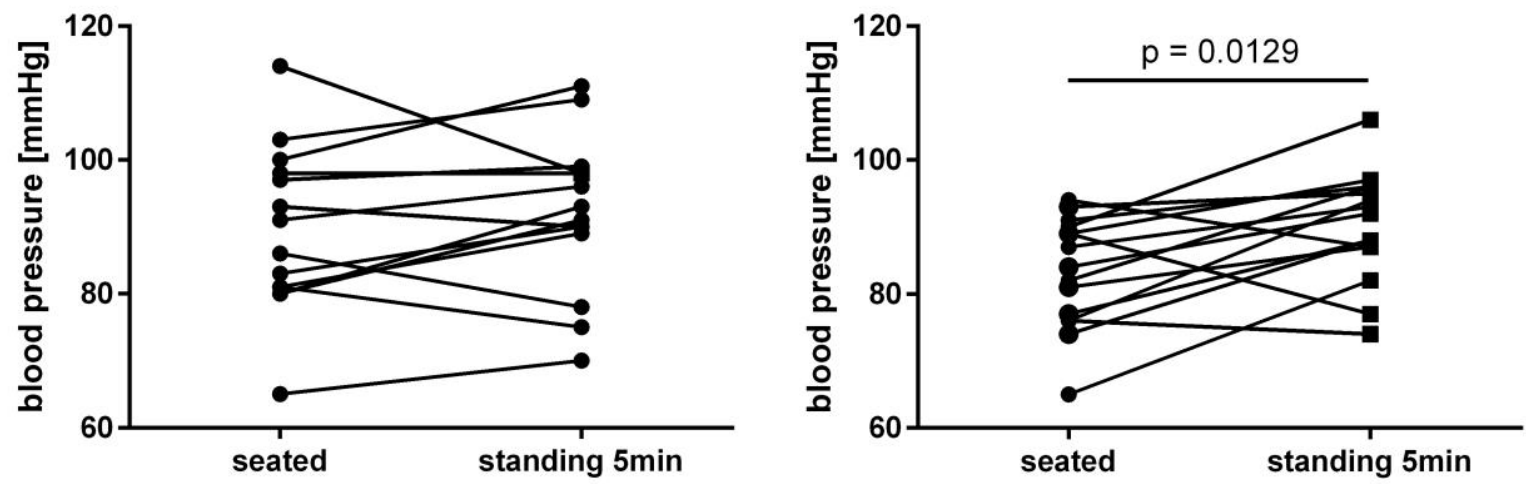

Fig S6 Sitting and standing heart rate and blood pressure

Heart rate and systolic and diastolic blood pressure sitting and after five minutes standing. Single values are shown in 14 female CCS/CFS and 15 female CCS patients. 
Figures
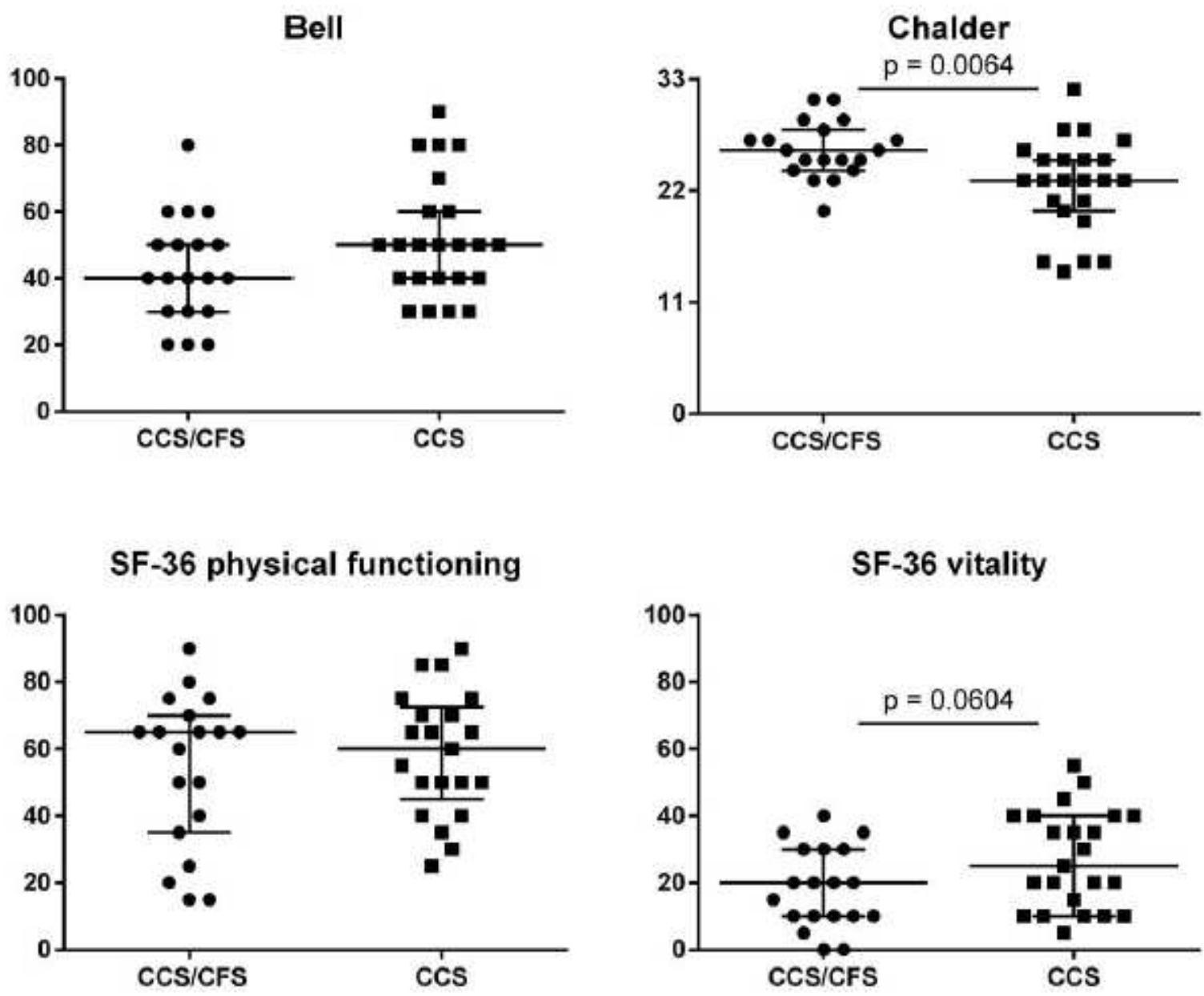

SF-36 role limitations due to physical health

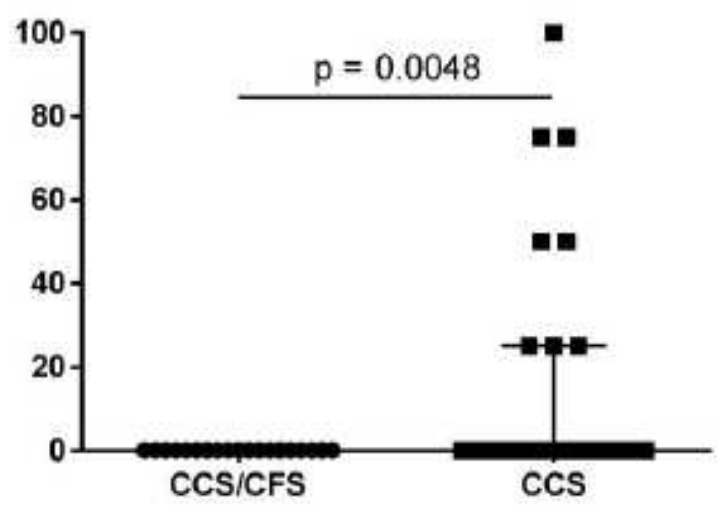

\section{SF-36 social functioning}

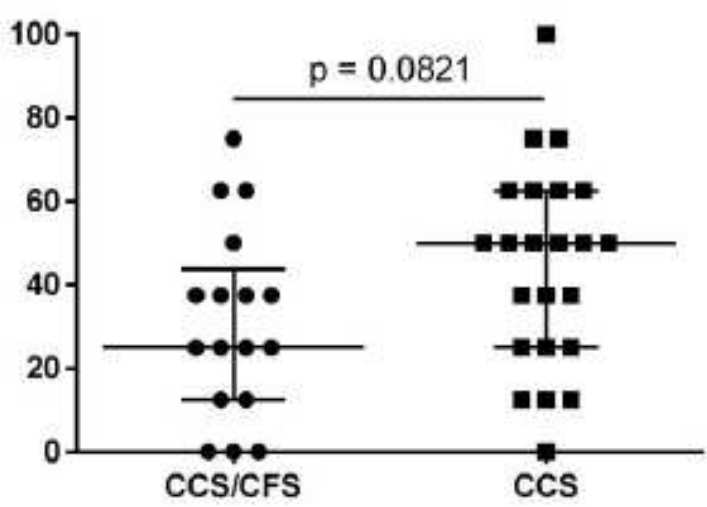

Figure 1

Severity of fatigue and disability. Bell disability score (score 0-100), Chalder fatigue score (score 0-33), and SF-36 physical function, vitality, role limitations, and social function (scores 0-100) in 19 CCS/CFS 
(17 for social functioning) and 23 CCS (21 for physical functioning) patients. Median, range, and single values are shown.
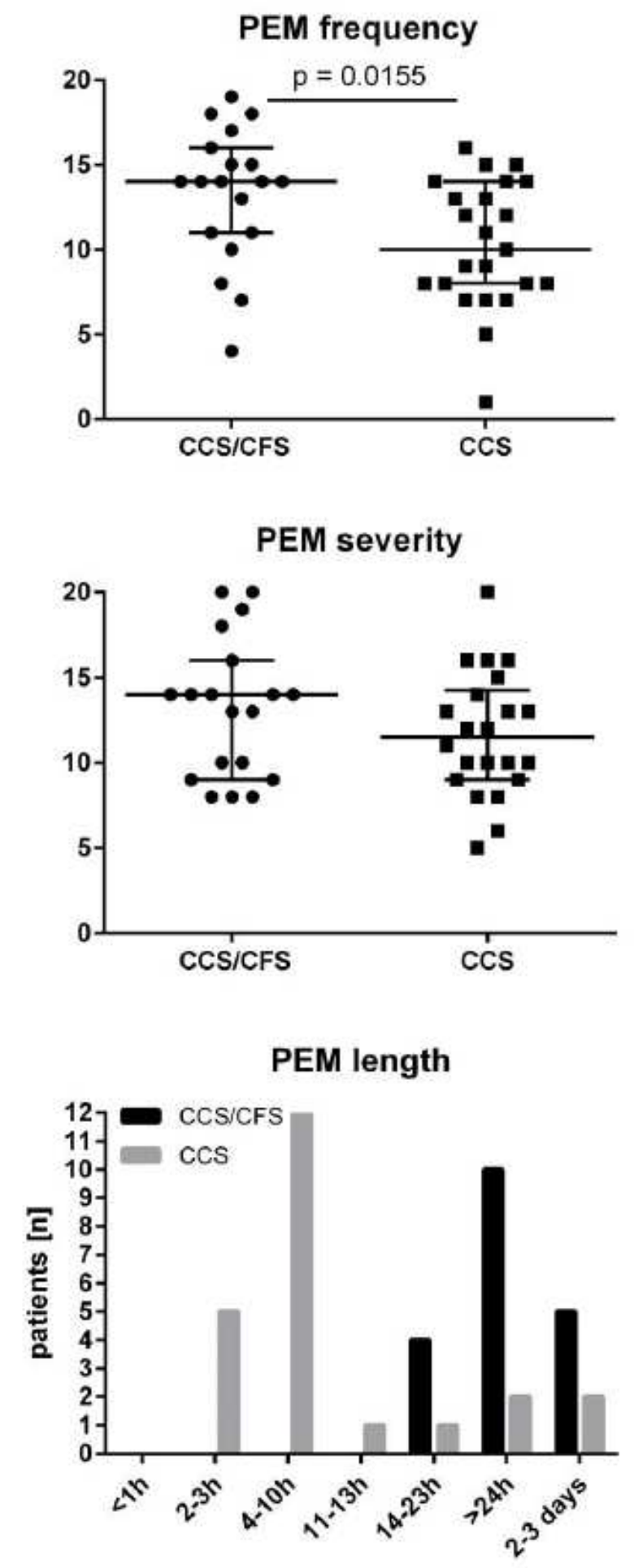

Figure 2

Frequency, severity and length of post exertional malaise (PEM). Frequency and severity of PEM was assessed on a five items scale with $0-20$ points (none to severest) and the length in seven categories 
(from $<1 \mathrm{~h}$ to $2-3$ days) according to Cotler. 9 Median, range, and single values are shown in 19 CCS/CFS and $23 / 22$ CCS patients (one patient completed only frequency block).
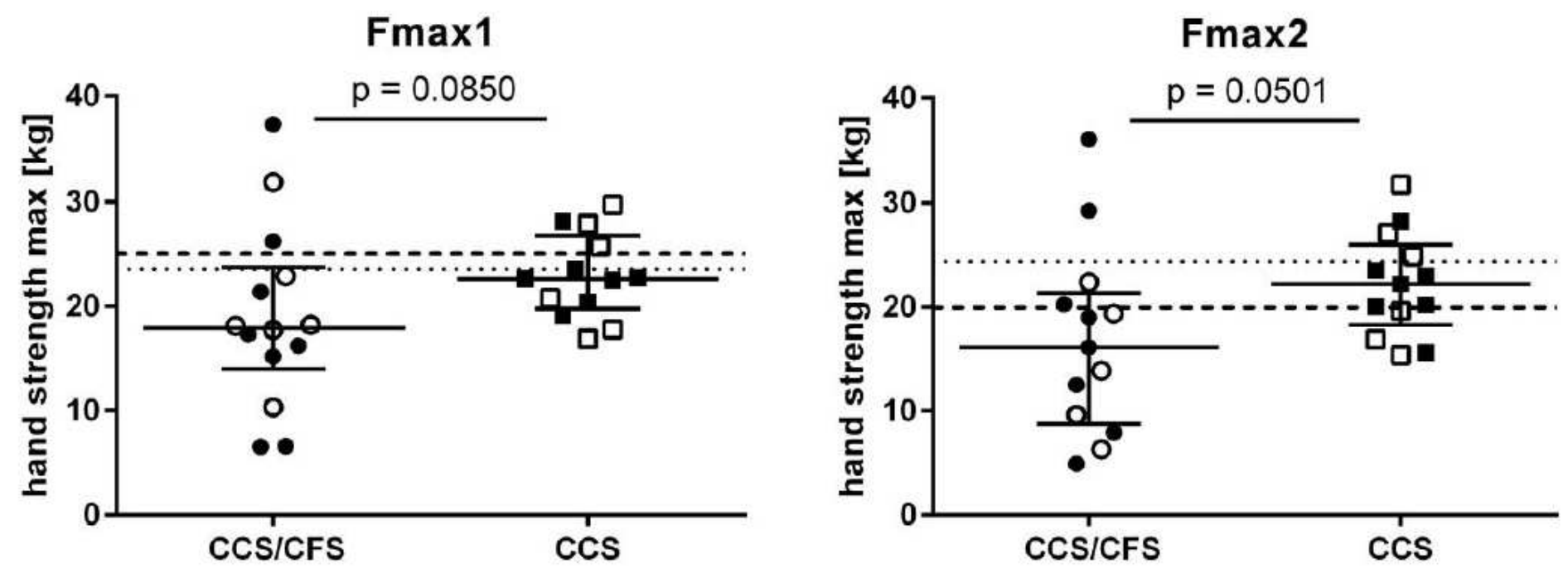

Fmean1
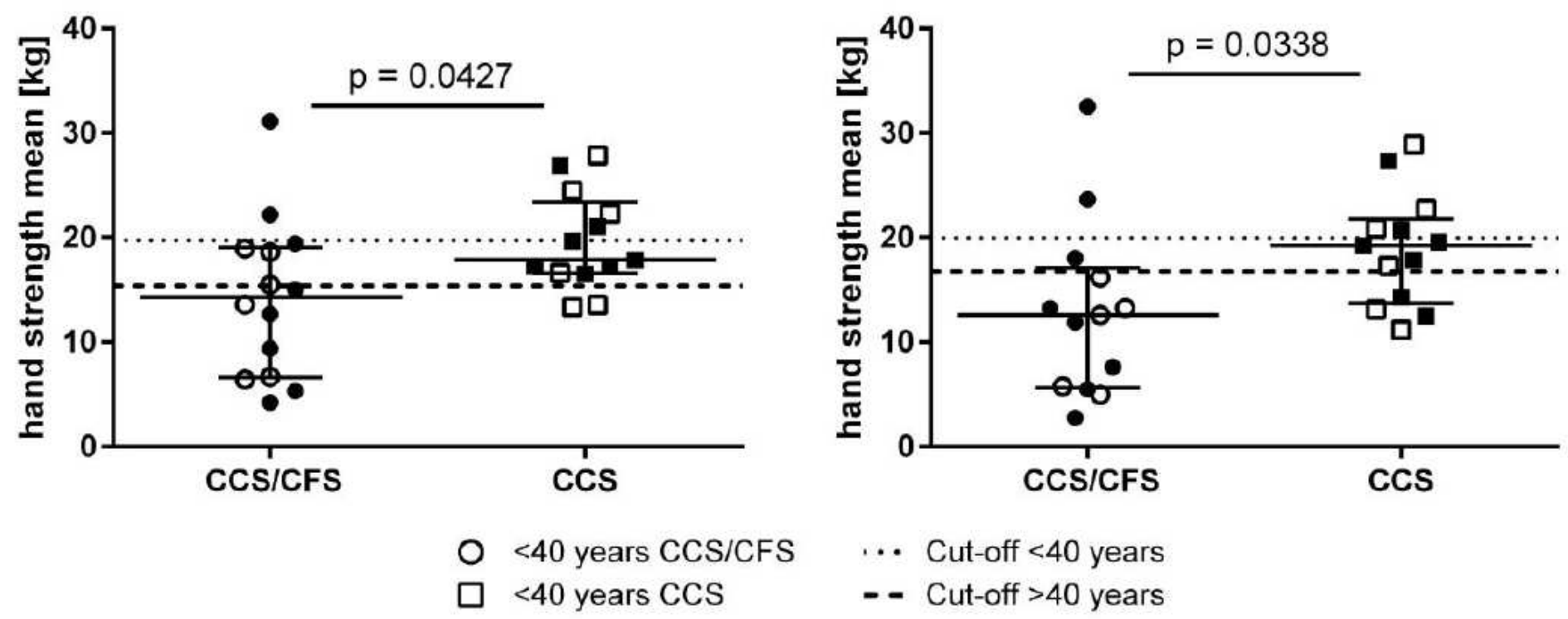

Figure 3

Hand grip strength (HGS). Fmax 1 and Fmean 1 of ten pulls in 14 female patients and repeat assessment after 60 minutes (Fmax2 and Fmean2 of ten pulls) in 13 female CCS/CFS and 13 female CCS patients are shown (median, range, and single values). Cut-off values of AUC reference values for age-matched healthy females are shown as dashed lines ( $<40$ years black dots and narrower dashed lines. $>40$ years white dots and wider dashed lines). 